\title{
Quantum algorithm for solving linear differential equations: Theory and experiment
}

\author{
Tao Xin,,${ }^{1,2, *}$ Shijie Wei, ${ }^{1,3, *}$ Jianlian Cui, ${ }^{4}$ Junxiang Xiao, ${ }^{1}$ Iñigo Arrazola, ${ }^{5}$ Lucas Lamata, ${ }^{5,6}$ Xiangyu Kong, ${ }^{1}$ Dawei Lu, ${ }^{2, \dagger}$ \\ Enrique Solano, ${ }^{5,7,8}$ and Guilu Long ${ }^{1,9,3, \$}$ \\ ${ }^{1}$ State Key Laboratory of Low-Dimensional Quantum Physics and Department of Physics, Tsinghua University, Beijing 100084, China \\ ${ }^{2}$ Shenzhen Institute for Quantum Science and Engineering and Department of Physics, Southern University of Science and Technology, \\ Shenzhen 518055, China \\ ${ }^{3}$ Beijing Academy of Quantum Information Sciences, Beijing 100193, China \\ ${ }^{4}$ Department of Mathematics, Tsinghua University, Beijing 100084, China \\ ${ }^{5}$ Department of Physical Chemistry, University of the Basque Country UPV/EHU, Apartado 644, 48080 Bilbao, Spain \\ ${ }^{6}$ Departamento de Física Atómica, Molecular y Nuclear, Universidad de Sevilla, 41080 Sevilla, Spain \\ ${ }^{7}$ IKERBASQUE, Basque Foundation for Science, Maria Diaz de Haro 3, 48013 Bilbao, Spain \\ ${ }^{8}$ International Center of Quantum Artificial Intelligence for Science and Technology (QuArtist) and Department of Physics, Shanghai \\ University, 200444 Shanghai, China \\ ${ }^{9}$ Tsinghua National Laboratory of Information Science and Technology and The Innovative Center of Quantum Matter, Beijing 100084, China
}

(Received 20 January 2020; accepted 12 February 2020; published 6 March 2020)

\begin{abstract}
Solving linear differential equations (LDEs) is a hard problem for classical computers, while quantum algorithms have been proposed to be capable of speeding up the calculation. However, they are yet to be realized in experiment as it cannot be easily converted into an implementable quantum circuit. Here, we present and experimentally realize an implementable gate-based quantum algorithm for efficiently solving the LDE problem: given an $N \times N$ matrix $\mathcal{M}$, an $N$-dimensional vector $b$, and an initial vector $x(0)$, we obtain a target vector $x(t)$ as a function of time $t$ according to the constraint $d x(t) / d t=\mathcal{M} x(t)+b$. We show that our algorithm exhibits an exponential speedup over its classical counterpart in certain circumstances, and a gate-based quantum circuit is produced which is friendly to the experimentalists and implementable in current quantum techniques. In addition, we experimentally solve a $4 \times 4$ linear differential equation using our quantum algorithm in a four-qubit nuclear magnetic resonance quantum information processor. Our algorithm provides a key technique for solving many important problems which rely on the solutions to linear differential equations.
\end{abstract}

DOI: 10.1103/PhysRevA.101.032307

\section{INTRODUCTION}

Linear differential equations (LDEs) are an important framework with which to describe the dynamics of a plethora of physical models, involving classical as well as quantum systems. They play key roles in many applications, e.g., predicting climate change and calculating fusion energy. In fact, many of the main applications of supercomputers are in the form of large systems of differential equations [1]. Generally, solving an LDE is a hard problem for a classical high-performance computer, in particular when the size of the configuration space is large, as for example in quantum systems or fluid dynamics.

A possible way to overcome the above difficulty is to utilize quantum computing. Quantum information processing is one of the most fruitful fields of research in physics nowadays. Besides the famous Shor factoring algorithm [2,3] and Grover search algorithm [4], a quantum computer is also capable of solving linear systems of equations [5,6] exponentially faster than any classical computers. In recent years, first steps

\footnotetext{
${ }^{*}$ These authors contributed equally to this work.

†ludw@sustech.edu.cn

†llong@tsinghua.edu.cn
}

towards solving linear equations have been demonstrated in optics [7,8], nuclear magnetic resonance (NMR) [9,10], and superconducting circuits [11]. However, extending the algorithm to differential equations is not straightforward. Although some quantum algorithms have been proposed [12-14], they are not easily implemented using state-of-theart techniques due to the lack of quantum circuits. Therefore, it is timely to design an implementable quantum algorithm and carry out the experimental demonstrations for solving LDEs in controllable quantum platforms.

In this work, we present a quantum algorithm for solving LDEs with the gate-based circuit only comprising of standard quantum gates, which is straightforward to be realized in current technologies. The precision of our algorithm can be boosted exponentially by adding the number of ancilla qubits. We further demonstrate it in a four-qubit NMR system, which is a quantum platform with a myriad of successes in the field of quantum technologies [15]. Many of the first demonstrations of quantum algorithms were achieved in this platform [16-23], which inherited a high degree of quantum control in NMR spectroscopy during the 20th century. This includes the recent demonstration of quantum machine learning [24] and linear solvers of equations [9]. In this work, we carry out a proof-of-principle experiment to implement an LDE solver in a four-qubit NMR quantum processor. 
Problem. The description of the problem for solving LDEs is as follows. An unknown vector $x(t)$ starts from an initial point $x(0)$ and follows an evolution described by an LDE $d x(t) / d t=\mathcal{M} x(t)+b$, where $\mathcal{M}$ is an arbitrary $N \times N$ matrix, while $b$ and $x(t)$ are $N$-dimensional vectors.

The analytical solution of the equation can be written as $x(t)=e^{\mathcal{M} t} x(0)+\left(e^{\mathcal{M} t}-I\right) \mathcal{M}^{-1} b$, involving matrix exponentials and inversions. For a classical computer, to approximate the solution in general requires time that scales at least as $O\left(N^{3}\right)$ [25], which is dominated by the computation of $e^{\mathcal{M} t}$. This runtime is not surprising since solving linear systems of equations, which is equivalent to computing the matrix inversion only, already requires $O(N)$ time classically [5]. Here, we provide a quantum algorithm to estimate the solution of such an equation in $O(\log N)$ time, which yields an exponential speedup compared to the classical counterpart in certain circumstances.

\section{OUR ALGORITHM FOR SOLVING LDES}

\section{A. The algorithm framework}

Now we present the basic idea of finding $x(t)$ based on our quantum algorithm. By Taylor expansion, the solution $x(t)$ is approximately

$$
x(t) \approx \sum_{m=0}^{k} \frac{(\mathcal{M} t)^{m}}{m !} x(0)+\sum_{n=1}^{k} \frac{\mathcal{M}^{n-1} t^{n}}{n !} b,
$$

where $k$ is the approximation order. The vectors $x(0)$ and $b$ can be described by quantum states $|x(0)\rangle=\sum_{j} x_{j}(0) /\|x(0)\||j\rangle$ and $|b\rangle=\sum_{j} b_{j} /\|b\||j\rangle$, respectively, where $x_{j}(0)$ and $b_{j}$ are the $j$ th elements of these vectors, $|j\rangle$ is the $N$-dimensional computational basis, and $\|\cdot\|$ is the modulus operation. The matrix $\mathcal{M}$ can be described by the operator $A$ defined as $A=\sum_{i, j} \mathcal{M}_{i j} /\|\mathcal{M}\||i\rangle\langle j|$. Hence, the $k$ th-order approximate solution converts to

$$
\begin{aligned}
|x(t)\rangle \approx & \sum_{m=0}^{k} \frac{\|x(0)\|(\|\mathcal{M}\| A t)^{m}}{m !}|x(0)\rangle \\
& +\sum_{n=1}^{k} \frac{\|b\|(\|\mathcal{M}\| A)^{n-1} t^{n}}{n !}|b\rangle,
\end{aligned}
$$

up to normalization. Our algorithm provides a general framework for computing Eq. (2) employing a quantum system with the assistance of ancilla qubits. The algorithm works for both unitary and nonunitary $A$ 's, and in the following we consider each of the two situations, respectively.

\section{Case I}

If the operator $A$ is unitary, the powers of $A$ will be also unitary. Let $U_{m}=A^{m}, U_{n}=A^{n}, C_{m}=\|x(0)\|(\|\mathcal{M}\| t)^{m} / m$ !, and $D_{n}=\|b\|(\|\mathcal{M}\| t)^{n-1} t / n$ !. By substituting them into Eq. (2), $x(t)$ can be represented by

$$
|x(t)\rangle \approx \frac{1}{\mathbb{N}^{2}}\left(\sum_{m=0}^{k} C_{m} U_{m}|x(0)\rangle+\sum_{n=1}^{k} D_{n} U_{n-1}|b\rangle\right),
$$

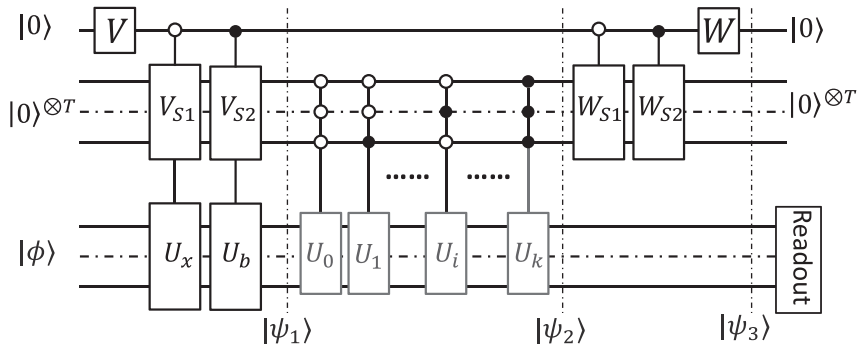

FIG. 1. Quantum circuit for solving LDEs when $A$ is unitary. It includes a first ancilla register with one qubit, a second ancilla register with $T=\log _{2}(k+1)$ qubits, and a work system. All ancilla registers are initially prepared in $|0\rangle|0\rangle^{T}$. The controlled operations $U_{x}$ and $U_{b}$ are used to create $|x(0)\rangle$ and $|b\rangle$, respectively. The evolution operator during encoding and decoding is $\sum_{\tau=0}^{k}|\tau\rangle\langle\tau| \otimes U_{\tau}$. The state after each step is denoted as $\left|\psi_{i}\right\rangle, i=1,2,3$. At the end of the circuit, we measure the state vector of the work system in the subspace where all ancilla qubits are $|0\rangle$.

where $\mathbb{N}^{2}=\mathcal{C}^{2}+\mathcal{D}^{2}$ with $\mathcal{C}=\sqrt{\sum C_{m}}$ and $\mathcal{D}=\sqrt{\sum D_{n}}$ is the normalization factor. Thus, the $j$ th element of $x(t)$ would be $x_{j}(t)=\mathbb{N}^{2}\langle j \mid x(t)\rangle$.

We employ a composite quantum system incorporating a work system and two ancilla registers to perform our algorithm as shown in Fig. 1. The framework is divided into four steps as follows.

a. Encoding. $\log _{2} N$ work qubits are needed to encode the $N$-dimensional vectors. $|x(0)\rangle$ and $|b\rangle$ are prepared and stored by the work qubits labeled by the subspace of the first ancilla register as $|0\rangle|x(0)\rangle$ and $|1\rangle|b\rangle$, respectively. In addition, a second ancilla register with $\log _{2}(k+1)$ qubits is added and transformed into a specific superposition state $|0\rangle \sum_{m=0}^{k} \sqrt{C_{m}}|m\rangle+|1\rangle \sum_{n=1}^{k} \sqrt{D_{n}}|n\rangle$.

Assume the input state of the work qubits is $|\phi\rangle$ and all ancilla qubits are $|0\rangle$ as shown in Fig. 1. The first operator $V$ is chosen as

$$
V=\frac{1}{\mathbb{N}}\left(\begin{array}{cc}
\mathcal{C} & \mathcal{D} \\
\mathcal{D} & -\mathcal{C}
\end{array}\right) .
$$

The encoded states $|x(0)\rangle$ and $|b\rangle$ are realized by performing controlled operations $U_{x}$ and $U_{b}$ on the input state $|\phi\rangle$ depending on the state of the first ancilla qubit, respectively. A joint-controlled operation $|0\rangle\left\langle 0\left|\otimes V_{S 1} \otimes U_{x}+\right| 1\right\rangle\langle 1| \otimes V_{S 2} \otimes$ $U_{b}$ is applied subsequently, where $U_{x}$ and $U_{b}$ are used to evolve the work qubits into $|x(0)\rangle$ and $|b\rangle$, and $V_{S 1}$ and $V_{S 2}$ are $(k+1) \times(k+1)$ operations acting on the second ancilla register. The elements of the first rows in $V_{S 1}$ and $V_{S 2}$ are chosen as

$$
\begin{aligned}
& V_{S 1}(:, 1)=1 / \mathcal{C}\left[\sqrt{C_{0}}, \sqrt{C_{1}}, \ldots, \sqrt{C_{k-1}}, \sqrt{C_{k}}\right], \\
& V_{S 2}(:, 1)=1 / \mathcal{D}\left[\sqrt{D_{1}}, \sqrt{D_{2}}, \ldots, \sqrt{D_{k}}, 0\right],
\end{aligned}
$$

while all other elements are arbitrary as long as $V_{S 1}$ and $V_{S 2}$ are unitary. After computation, the initial state $\left|\psi_{\text {in }}\right\rangle=|0\rangle \otimes$ $|0\rangle^{\otimes T} \otimes|\phi\rangle$ is evolved into

$\left|\psi_{1}\right\rangle=\frac{1}{\mathbb{N}}\left(|0\rangle \sum_{m=0}^{k} \sqrt{C_{m}}|m\rangle|x(0)\rangle+|1\rangle \sum_{n=1}^{k} \sqrt{D_{n}}|n-1\rangle|b\rangle\right)$. 
$b$. Entanglement creation. A series of controlled operations is applied, to realize the operation $\sum_{\tau=0}^{k}|\tau\rangle\langle\tau| \otimes U_{\tau}$ on the work qubits which is controlled by the second ancilla register. The work qubits and the ancilla registers are now entangled, and the whole state is

$$
\begin{aligned}
\left|\psi_{2}\right\rangle= & \frac{1}{\mathbb{N}}\left(|0\rangle \sum_{m=0}^{k} \sqrt{C_{m}}|m\rangle U_{m}|x(0)\rangle\right. \\
& \left.+|1\rangle \sum_{n=1}^{k} \sqrt{D_{n}}|n-1\rangle U_{n-1}|b\rangle\right) .
\end{aligned}
$$

c. Decoding. All the operations in the encoding stage are reversely applied. $|0\rangle\left\langle 0\left|\otimes W_{S 1}+\right| 1\right\rangle\langle 1| \otimes W_{S 2}$ on the ancilla registers are applied, where $W_{S 1}=V_{S 1}^{\dagger}$ and $W_{S 2}=V_{S 2}^{\dagger}$, followed by the last operator $W=V^{\dagger}$ on the first ancilla. Only the subspace where all ancilla qubits are $|0\rangle$ is concerned, and the state of the whole system in this subspace is

$$
\left|\psi_{3}\right\rangle=\frac{1}{\mathbb{N}^{2}}|0\rangle|0\rangle{ }^{\otimes T}\left(\sum_{m=0}^{k} C_{m} U_{m}|x(0)\rangle+\sum_{n=1}^{k} D_{n} U_{n-1}|b\rangle\right) .
$$

d. Measurement. We measure the final state of the work qubits in the subspace where all ancilla qubits are $|0\rangle$. It is obvious by comparing with Eq. (3) that $|x(t)\rangle$ will be directly extracted; i.e., the solution to the LDE is obtained up to a factor $\mathbb{N}^{2}$.

\section{Case II}

This case in which $A$ is nonunitary is similar to the first case, but more complicated. As $A$ can be decomposed into a linear combination of unitary operators $A=\sum_{i=1}^{L} \alpha_{i} A_{i}$, as given in the duality quantum computing formalism [26-29], we need a third ancilla register to label the linear combination $A_{i}$ 's. Compared with the first case, we need more ancilla qubits and controlled operations. We give details in Appendix A.

\section{B. The complexity of our algorithm}

There is no phase estimation or quantum Fourier transformation in our algorithms. The speedup of the algorithm comes from the nonunitary [see Eq. (2)] to unitary transformation by adding ancillary qubits. The combination of an ancillary system with a work system provides additional freedom to process information. We create superposition states on the ancillary system, and then perform controlled operations on the work system. The physical picture is that different unitary operations are implemented simultaneously on the work system but in the different subspaces. Subsequently, all the operations in the first stage are reversely applied. The physical picture is that we combine all the information in the different subspaces and obtain a final result in a subspace where the ancillary system is $|0\rangle$. In fact, superposition and entanglement, as the most important quantum resources, are both employed during the algorithm as shown in Fig. 1. Hence, we deem it natural to yield quantum speedup in this algorithm for solving special problems.

Generally speaking, the cost of a quantum algorithm involves two aspects. One aspect is qubit resources corresponding to space complexity. The other aspect is gate complexity
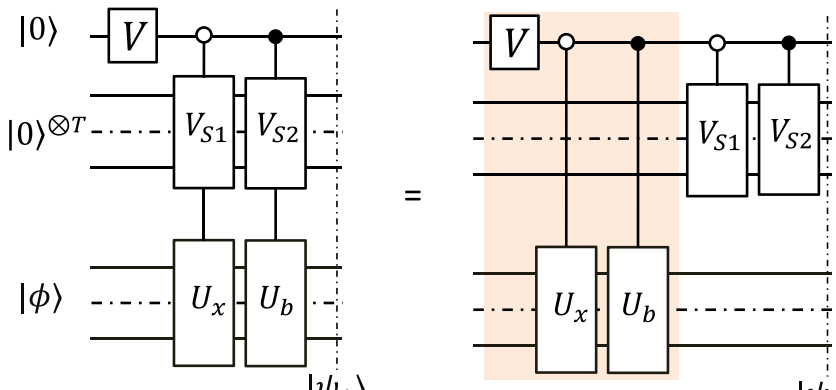

$\left|\psi_{1}\right\rangle$

$\left|\psi_{1}\right\rangle$

FIG. 2. Quantum circuit in the encoding part for preparing the initial state $\left|\psi_{1}\right\rangle$ when matrix $A$ is unitary.

corresponding to time complexity. Next, we present a detailed discussion about complexity for our algorithm when $A$ is unitary.

Qubit resources. In our algorithm, we need ancillary qubits to realize the nonunitary evolution in Eq. (2) in a unitary way. The number of ancilla qubits is $1+\log (k+1)$, where $k$ is the approximate order in Eq. (2) and determines the gap $\epsilon$ between the ideal and approximate solutions with $k=\ln \left(C_{0} / \epsilon\right)$ and $C_{0}$ constant (proof in Appendix B). In most cases, a fairly small $k$ is sufficient, as it exponentially improves the solution's precision $\epsilon$.

Gate complexity. In the encoding part (before $\left|\psi_{1}\right\rangle$ in the circuit shown in Fig. 1), it is equivalent to a circuit where the four controlled operations $U_{x}, U_{b}, V_{S 1}$, and $V_{S 2}$ are implemented subsequently (see Fig. 2). Let us analyze the gate complexity in the encoding part block by block.

For the system, the vectors $x(0)$ and $b$ are first loaded into the initial state $|0\rangle|x(0)\rangle$ and $|1\rangle|b\rangle$ (controlled $U_{x}$ and $U_{b}$ ). There are some proposed methods for loading the classical data, such as the quantum random access memory (qRAM) approach [30-32]. qRAM is an important approach to prepare the initial state in many algorithms, especially in quantum machine learning. The complexity for updating the data is about $O(\log N)$ after quantum memory is established. Hence, the complexity to prepare the $C|0\rangle|x(0)\rangle+D|1\rangle|b\rangle$ (orange part) is $O(\log (2 N)) \sim O(\log N)$ in Fig. 2. The controlled operators $V_{S 1}$ and $V_{S 2}$ are real $(2 k+2) \times(2 k+2)$ matrices. They can be decomposed into $O\left((\log (2 k+2))^{3} \times(2 k+2)^{2}\right) \sim O\left(k^{2}\right)$ elementary gates via QR decomposition [33]. Thus, the gate complexity for preparing the initial state $\left|\psi_{1}\right\rangle$ is $O(\log N+$ $k^{2}$ ) by ignoring smaller terms and constants.

In the middle part of Fig. 1, the controlled operations $U_{m}$ can be decomposed into $O((k+1) \log (k+1) \log N) \sim$ $O(k \log k \log N)$ basic gates [34].

In the decoding part, all the operations in the encoding stage are reversely applied. Thus, it has the same gate complexity compared to the encoding part.

In summary, the gate complexity of our algorithm is $O\left(k \log k \log N+\log N+k^{2}\right)$. The performance of our algorithm depends on two factors, $k$ and $N$. If $k$ is polylogarithmic in $N$, our algorithm achieves an exponential speedup. In most cases, a fairly small Taylor order $k$ is enough, as it exponentially improves the solution's precision. Therefore, we conclude that our algorithm yields a quantum speedup in most circumstances. The complexity analysis for nonunitary 
(a)

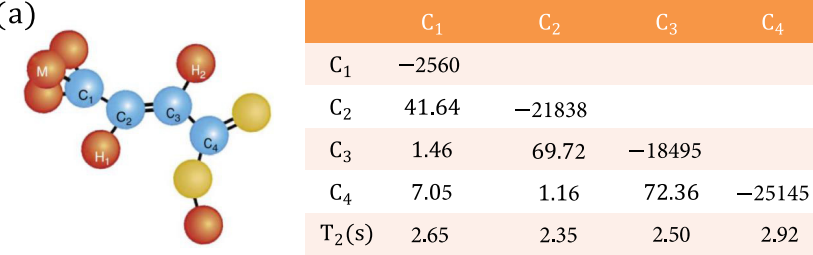

(b)

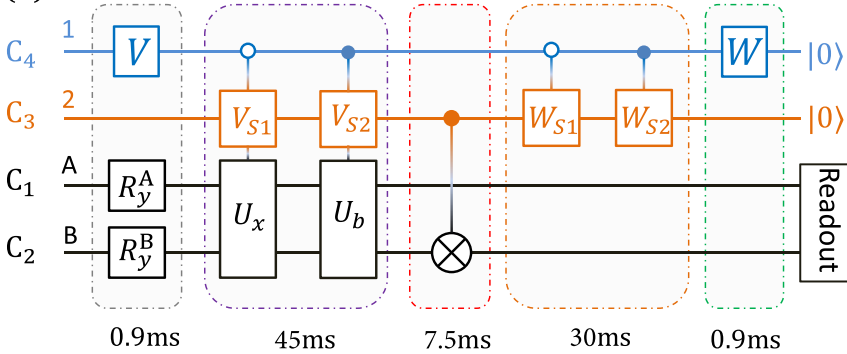

FIG. 3. (a) Molecular structure and Hamiltonian parameters of ${ }^{13} \mathrm{C}$-labeled trans-crotonic acid. $\mathrm{C}_{1}, \mathrm{C}_{2}, \mathrm{C}_{3}$, and $\mathrm{C}_{4}$ are used as four qubits in the experiment, while $\mathrm{M}, \mathrm{H}_{1}$, and $\mathrm{H}_{2}$ are decoupled throughout the experiment. In the table, the chemical shifts with respect to the Larmor frequency and J-coupling constants (in hertz) are listed by the diagonal and off-diagonal numbers, respectively. The relaxation time scales $T_{2}$ (in seconds) are shown at the bottom. (b) NMR quantum circuit to realize the solution of a four-dimensional LDE with four qubits. A (labeled by $\mathrm{C}_{1}$ ) and $\mathrm{B}$ (labeled by $\mathrm{C}_{2}$ ) are work qubits to encode the vectors $|x(0)\rangle$ and $|b\rangle$. Qubit 1 (labeled by $\mathrm{C}_{4}$ ) and qubit 2 (labeled by $\mathrm{C}_{3}$ ) are used as ancilla qubits. This circuit starts from $|0000\rangle$ which is prepared by the spatial average method. The input state $|\phi\rangle$ is then created by implementing the rotations $R_{y}^{\mathrm{A}}(\beta)$ and $R_{y}^{\mathrm{B}}(\beta)$ on the work qubits. $U_{x}=I \otimes I$ and $U_{b}=\sigma_{x} \otimes \sigma_{x}$ are applied to realize the preparation of the vectors $|x(0)\rangle$ and $|b\rangle$, respectively. Finally, we measure the state of the work qubits when the ancilla qubits are $|00\rangle$. Durations of the optimized pulses for each step are also given.

$A$ is presented in Appendix C. We also present an alternative approach of our algorithm to solve LDEs with the less ancillary qubits in Appendix D.

\section{EXPERIMENTAL IMPLEMENTATION}

\section{A. Experimental setup}

Experimentally, we demonstrate the quantum algorithm for solving a four-dimensional LDE with a four-qubit nuclear magnetic resonance system. We make use of the nuclear spins in a sample of ${ }^{13} \mathrm{C}$-labeled trans-crotonic acid dissolved in acetone- $\mathrm{D}_{6}$. The internal Hamiltonian of this system can be described as

$$
\mathcal{H}_{\text {int }}=\sum_{j=1}^{4} \pi v_{j} \sigma_{z}^{j}+\sum_{j<k,=1}^{4} \frac{\pi}{2} J_{j k} \sigma_{z}^{j} \sigma_{z}^{k},
$$

where $v_{j}$ is the chemical shift of the $j$ th spin and $J_{j k}$ is the $J$-coupling strength between spins $j$ and $k$. We assigned $\mathrm{C}_{1}$ and $\mathrm{C}_{2}$ as system qubits and $\mathrm{C}_{4}$ and $\mathrm{C}_{3}$ as ancilla qubits, respectively. The structure and parameters of the molecule are shown in Fig. 3(a). All experiments were carried out on a Bruker ADVANCE $600 \mathrm{MHz}$ spectrometer at room temperature.

\section{B. Protocol and results}

In experiment, we demonstrate a four-dimensional LDE $d x(t) / d t=\mathcal{M} x(t)+b$ with

$$
\begin{aligned}
\mathcal{M} & =I \otimes I+2 I \otimes \sigma_{x}, \\
x(0) & =\left[\cos ^{2} \frac{\beta}{2}, \cos \frac{\beta}{2} \sin \frac{\beta}{2}, \cos \frac{\beta}{2} \sin \frac{\beta}{2}, \sin ^{2} \frac{\beta}{2}\right], \\
b & =\left[\sin ^{2} \frac{\beta}{2}, \cos \frac{\beta}{2} \sin \frac{\beta}{2}, \cos \frac{\beta}{2} \sin \frac{\beta}{2}, \cos ^{2} \frac{\beta}{2}\right] .
\end{aligned}
$$

The value of $\beta$ ranges from $0.1 \pi$ to $0.5 \pi$ with the increment $0.1 \pi$. The nonunitary $\mathcal{M}$ can be decomposed into a linear combination of $\mathcal{M}_{0}=I \otimes I$ and $\mathcal{M}_{1}=I \otimes \sigma_{x}$. The initial vector $|x(0)\rangle$ and $|b\rangle$ are realized by applying two-qubit operations $U_{x}$ and $U_{b}$ on $|\phi\rangle$, respectively, where $|\phi\rangle$ is created by $|\phi\rangle=R_{y}^{\mathrm{A}}(\beta) R_{y}^{\mathrm{B}}(\beta)|00\rangle$ with $R_{y}^{j}(\beta)=e^{-i \beta \sigma_{y}^{j} / 2}$.

The accuracy of the solution depends on the order $k$. We set the order $k=4$, leading to four qubits to implement the quantum circuit [see Fig. 3(c)] for solving the LDE. The forms of $V, W, U_{c}, V_{S 1}, V_{S 2}, W_{S 1}$, and $W_{S 2}$ can be found in Appendix E. To experimentally realize our algorithm, we make use of the nuclear spins in a sample of ${ }^{13} \mathrm{C}$-labeled trans-crotonic acid dissolved in acetone- $\mathrm{D}_{6}$ shown in Fig. 3(a) [35-37].

First, we use the spatial averaging technique to prepare the pseudopure state (PPS) $[38,39]$ from the thermal equilibrium. At thermal equilibrium, an NMR sample stays in the Boltzmann distribution,

$$
\rho_{\text {thermal }}=\frac{\mathcal{I}}{16}+\epsilon\left(\sigma_{z}^{1}+\sigma_{z}^{2}+\sigma_{z}^{3}+\sigma_{z}^{4}\right),
$$

where $\mathcal{I}$ is a $16 \times 16$ identity matrix and the polarization $\epsilon \approx 10^{-5}$. It is a highly mixed state which is not suitable for quantum computing. Starting from this state, we use the spatial averaging technique to realize the preparation of the following PPS:

$$
\rho_{0000}=\frac{1-\epsilon}{16} \mathcal{I}+\epsilon|0000\rangle\langle 0000| .
$$

The initialization processing usually includes local unitary rotations and $z$-gradient fields for suppressing the undesired coherence. Considering that the identity part does not evolve under any unitary operations or influence any measurements in NMR, the deviation density matrix $|0000\rangle\langle 0000|$ can serve as the initial state of the quantum circuit. Figure 4 presents experimental spectra of the PPS for different carbon nuclei and the reconstructed density matrix of the PPS by performing quantum state tomography. The fidelity between the ideal pure state $|0000\rangle$ and the experimental PPS is about 0.99 , which underpins subsequent experiments.

Subsequently, we perform the operations involved in our algorithm. All the operations are individually realized using shaped pulses optimized by the gradient method [40-42]. Each shaped pulse is simulated to be about 0.998 fidelity while being robust to the static field distributions and inhomogeneity.

Finally, we need to measure the state of the work qubits when the ancilla are $|00\rangle$. In experiment, we performed fourqubit state tomography to extract the desired results from the final density matrix $[43,44]$. It also enables us to evaluate the 

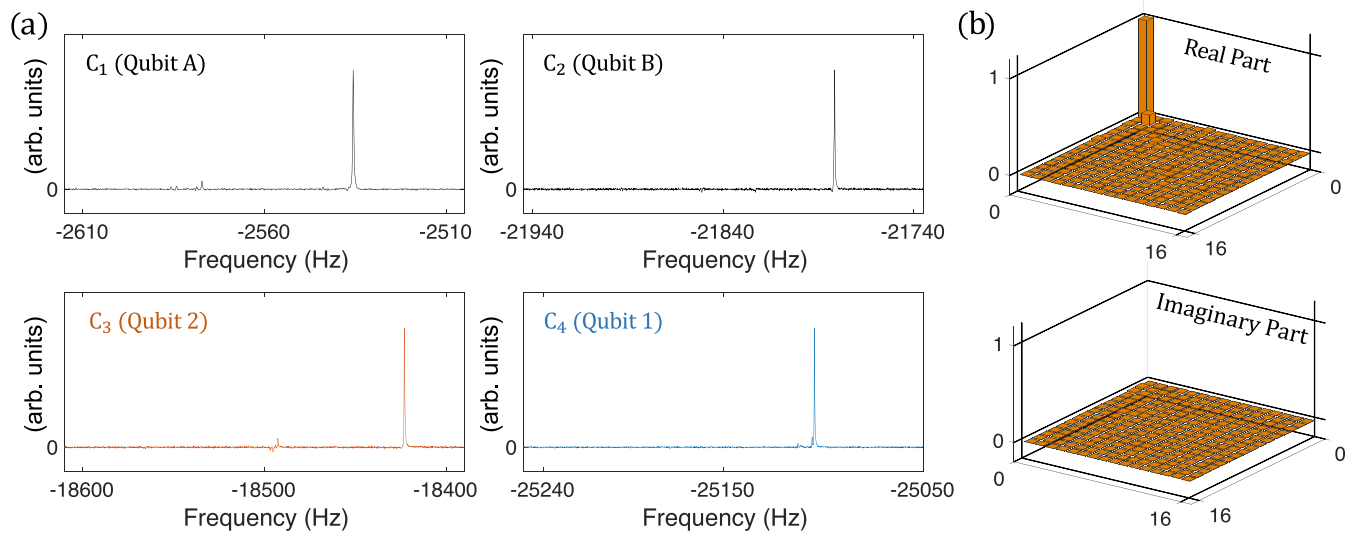

FIG. 4. Experimental spectra of the nuclei $C_{1}-C_{4}$ and the reconstructed density matrix of the PPS. (a) NMR signals of the nuclei $C_{1}-C_{4}$ are measured by applying the corresponding $\pi / 2$ readout pulses after the PPS preparation. (b) Top and bottom plots respectively show the real and imaginary part of the reconstructed PPS matrix. The $z$ axis represents the value of the element in the matrix.

quality of our implementation by comparing the distance between the target state $\rho_{t h}$ and the experimentally reconstructed density matrix $\rho_{\text {expt }}$.

We fix $t=0.4$ and range $\beta$ from $0.1 \pi$ to $0.5 \pi$ with the increment $0.1 \pi$ in experiments. In other words, we demonstrate the solutions to five LDEs with different initial vectors $|x(0)\rangle$ and offset vectors $|b\rangle$ at a fixed time $t=0.4$. For each value of $\beta$, the experiment is repeated four times to estimate the uncertainty. After the implementation of the quantum circuit, we perform the four-qubit state tomography by applying 17 readout pulses (Appendix F). On average, the experimental fidelity for all states is $\bar{F}=0.946(4)$, estimated by

$$
F\left(\rho_{t h}, \rho_{\text {expt }}\right)=\operatorname{Tr}\left(\rho_{t h} \rho_{\text {expt }}\right) / \sqrt{\operatorname{Tr}\left(\rho_{t h}^{2}\right) \operatorname{Tr}\left(\rho_{\text {expt }}^{2}\right)} .
$$

Taking $\beta=0.1 \pi$ as an example, the comparison between the experimental and simulated NMR spectra of the work qubits is given in Figs. 5(a) and 5(b), and they are in excellent agreement. The real parts of the density matrices for $\rho_{\text {expt }}$ and $\rho_{t h}$ are also displayed in Fig. 5(c) to evaluate the performance
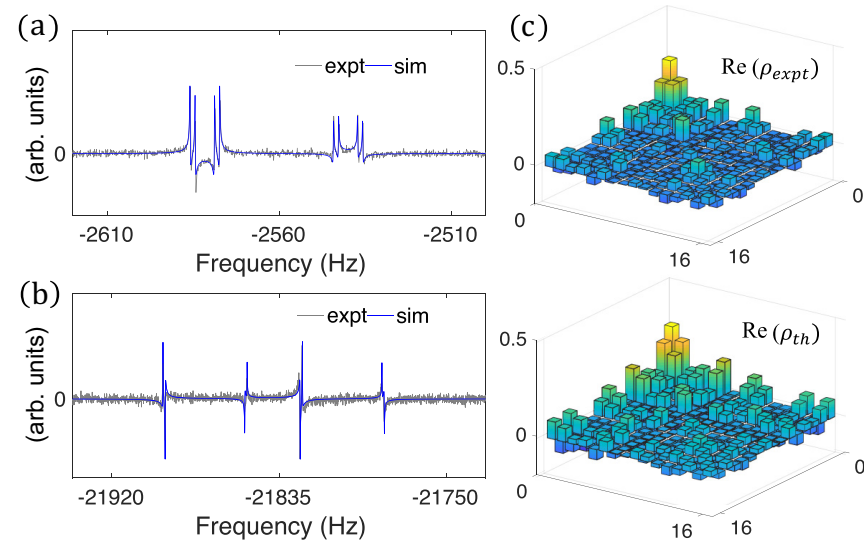

FIG. 5. NMR spectra of (a) $\mathrm{C}_{1}$ (work qubit A) and (b) $\mathrm{C}_{2}$ (work qubit B) followed by a readout pulse $R_{y}^{12}(\pi / 2) R_{x}^{\mathrm{AB}}(\pi / 2)$ for $\beta=$ $0.1 \pi$. The gray and blue lines show the experimental and simulated spectra, respectively. (c) Real part of the density matrices $\rho_{\text {expt }}$ and $\rho_{t h}$ for $\beta=0.1 \pi$. of our experiment. Furthermore, considering that $\mathcal{M},|x(0)\rangle$, and $|b\rangle$ are all real in our setting, the solution $x(t)$ should be also real. We use a maximum-likelihood (ML) approach to construct a real state $\rho_{m l}$ which is closest to the experimentally measured density matrix $\rho_{\text {expt }}[35,45]$. After obtaining $\rho_{m l}$, we calculate the reduced state vector of work qubits $A$ and $B$ in the subspace where the ancilla are $|00\rangle$, and then reproduce the solutions to the LDEs by amplifying the result by $\mathbb{N}^{2}=$ 4.059. Table I summarizes all experimental results of the five LDEs and the comparison between theory and experiment.

\section{CONCLUSION}

In summary, we present a quantum algorithm and the relevant quantum circuit for solving $N$-variable LDEs, which achieves an exponential speedup $O(\log N)$ over its classical counterpart in certain circumstances. As a proof-of-principle demonstration, we experimentally realize the solution to a set of LDEs with the dimension $4 \times 4$ in a four-qubit NMR quantum processor. The experimental solutions to these LDEs are obtained with about $5.36 \%$ infidelity. The error mainly comes from the imperfections of the initial state preparation, the imprecisions and inhomogeneity of the optimized pulses, the decoherence effect, and the readout error in tomography. We numerically simulate each of the above factors to estimate the error distribution (Appendix G). On average, numerical results show that the error in state preparation, shaped pulses, decoherence, and readout are $1.07 \%, 0.84 \%, 1.19 \%$, and $1.23 \%$, respectively. The sum is $4.33 \%$ by assuming all errors are additive, which is slightly smaller than the measured $1-\bar{F}$. As the discrepancy is quite small, our error estimation is consistent with the experimental results. It indicates the accuracy of the experimental implementation.

Unlike Shor's or Harrow-Hassidim-Lloyd (HHL) algorithms where the core is phase estimation, the speedup of our algorithm comes from the nonunitary [see Eq. (2)] to unitary transformation by adding ancillary qubits. It is called the linear combination of unitaries (LCU). LCU is also a universal subroutine in designing and developing quantum algorithms [46]. Traditional quantum computing based on a closed quantum system undergoes unitary evolution. 
TABLE I. Experimental results of our algorithm for solving an LDE $d x(t) / d t=\mathcal{M} x(t)+b$ at a fixed time $t=0.4 \mathrm{~s}$. $\beta$ (see Fig. 3) ranges from $0.1 \pi$ to $0.5 \pi$ with a $0.1 \pi$ increment. Theoretical and experimental solutions $x(t)$ are both shown. To evaluate the performance, we compute the inner product (normalized) between the theoretical and experimental $x(t)$. Similarity between vectors $\mathbf{c}$ and $\mathbf{d}$ is defined as the cosine similarity $|\mathbf{c} \cdot \mathbf{d}| /|\mathbf{c}|^{2}|\mathbf{d}|^{2}$. Error bars come from the uncertainty in repeated experiments, which is mainly attributed to the drift of temperature and inhomogeneity of the magnetic field.

\begin{tabular}{|c|c|c|c|c|c|c|c|c|c|c|}
\hline \multirow[b]{2}{*}{$\beta$} & \multicolumn{2}{|r|}{$0.1 \pi$} & \multicolumn{2}{|r|}{$0.2 \pi$} & \multicolumn{2}{|r|}{$0.3 \pi$} & \multicolumn{2}{|r|}{$0.4 \pi$} & \multicolumn{2}{|r|}{$0.5 \pi$} \\
\hline & Theory & Experiment & Theory & Experiment & Theory & Experiment & Theory & Experiment & Theory & Experiment \\
\hline \multirow[t]{4}{*}{ Results $x(t)$} & 2.184 & $2.280 \pm 0.006$ & 2.295 & $2.333 \pm 0.005$ & 2.305 & $2.277 \pm 0.003$ & 2.214 & $2.154 \pm 0.002$ & 2.030 & $2.066 \pm 0.006$ \\
\hline & 1.676 & $1.747 \pm 0.009$ & 1.951 & $2.059 \pm 0.006$ & 2.110 & $2.193 \pm 0.002$ & 2.137 & $2.214 \pm 0.006$ & 2.030 & $2.049 \pm 0.004$ \\
\hline & 0.635 & $0.708 \pm 0.007$ & 1.066 & $1.040 \pm 0.008$ & 1.466 & $1.453 \pm 0.004$ & 1.799 & $1.829 \pm 0.005$ & 2.030 & $2.105 \pm 0.003$ \\
\hline & 0.819 & $0.881 \pm 0.008$ & 1.134 & $1.064 \pm 0.007$ & 1.462 & $1.372 \pm 0.007$ & 1.770 & $1.701 \pm 0.002$ & 2.030 & $1.892 \pm 0.004$ \\
\hline Similarity & \multicolumn{2}{|c|}{$99.99 \% \pm 0.003 \%$} & \multicolumn{2}{|c|}{$99.93 \% \pm 0.009 \%$} & \multicolumn{2}{|c|}{$99.94 \% \pm 0.003 \%$} & \multicolumn{2}{|c|}{$99.95 \% \pm 0.003 \%$} & \multicolumn{2}{|c|}{$99.92 \% \pm 0.005 \%$} \\
\hline
\end{tabular}

However, the evolution for this LDE problem described by Eq. (2) is nonunitary. To make it implementable in a unitary quantum circuit, we added an ancillary system to let the evolution of the whole system be unitary and provided the gate-based quantum circuit which is friendly to experimentalists. This is the intuition when developing our algorithm. Furthermore, as long as the target is some linear combination of unitary operators, our model provides a general way to enable its realization in a unitary quantum circuit at the cost of ancillary qubits. We anticipate it to be heuristic when exploring quantum algorithms with nonunitary evolutions in the future, such as route optimization of unmanned vehicles in artificial intelligence.

\section{ACKNOWLEDGMENTS}

S.W. and G.L. are grateful to the following funding sources: National Basic Research Program of China, the National Natural Science Foundation of China (Grants No. 11974205 and No. 11774197), the National Key Research and Development Program of China (2017YFA0303700), the Key Research and Development Program of Guangdong province (2018B030325002), and Beijing Advanced Innovation Center for Future Chip (ICFC). T.X. and D.L. are supported by the National Natural Science Foundation of China (Grants No. 11905099, No. 11605005, No. 11875159, and No. U1801661), Science, Technology and Innovation Commission of Shenzhen Municipality (Grants No. ZDSYS20170303165926217 and No. JCYJ20170412152620376), Guangdong Innovative and Entrepreneurial Research Team Program (Grant No. 2016ZT06D348), and Guangdong Basic and Applied Basic Research Foundation (Grant No. 2019A1515011383). I.A., L.L., and E.S. acknowledge financial support from Spanish Government PGC2018-095113-B-I00 (MCIU/AEI/FEDER, UE), EU FET Open Grant Quromorphic (828826), Basque Government IT986-16, Ph. D. Grant No. PRE-2015-1-0394, and the projects QMiCS (820505) and OpenSuperQ (820363) of the EU Flagship on Quantum Technologies.

\section{APPENDIX A: MATHEMATICAL DETAILS OF OUR ALGORITHM}

We present a mathematical representation of our algorithm by considering the following two cases.

\section{1. $A$ is unitary}

In order to solve an LDE where the matrix $A$ is unitary, we need a composite quantum system with a $(1+T)$-qubit ancilla register and a $\log _{2}(N)$-qubit work system. Suppose the input state of the work system is $|\phi\rangle$ and all ancilla qubits are prepared in state $|0\rangle|0\rangle^{\otimes T}$. First, the first ancilla qubit evolves to a superposition state after a unitary operation $V$ is performed,

$$
V=\frac{1}{\mathbb{N}}\left(\begin{array}{cc}
\mathcal{C} & \mathcal{D} \\
\mathcal{D} & -\mathcal{C}
\end{array}\right) .
$$

The encoded states $|x(0)\rangle$ and $|b\rangle$ are realized by performing controlled operations $U_{x}$ and $U_{b}$ on the input state $|\phi\rangle$, respectively. The initial state $|0\rangle|0\rangle^{\otimes T}|\phi\rangle$ is thus

$$
\frac{\mathcal{C}}{\mathbb{N}}|0\rangle|0\rangle^{\otimes T}|x(0)\rangle+\frac{\mathcal{D}}{\mathbb{N}}|1\rangle|0\rangle^{\otimes T}|b\rangle .
$$

Then, we define $(k+1) \times(k+1)$ controlled operations $V_{S 1}$ and $V_{S 2}$ with

$$
\begin{aligned}
V_{S 1} & =\frac{1}{\mathcal{C}}\left(\begin{array}{llllll}
\sqrt{C_{0}} & Q & Q & Q & Q & Q \\
\sqrt{C_{1}} & Q & Q & Q & Q & Q \\
\cdots & Q & Q & Q & Q & Q \\
\sqrt{C_{k}} & Q & Q & Q & Q & Q
\end{array}\right)_{(k+1) \times(k+1)},\left(A^{2}\right) \\
V_{S 2}= & \frac{1}{\mathcal{D}}\left(\begin{array}{cccccc}
\sqrt{D_{1}} & Q & Q & Q & Q & Q \\
\sqrt{D_{2}} & Q & Q & Q & Q & Q \\
\cdots & Q & Q & Q & Q & Q \\
\sqrt{D_{k}} & Q & Q & Q & Q & Q \\
0 & Q & Q & Q & Q & Q
\end{array}\right)_{(k+1) \times(k+1)},(A 4)
\end{aligned}
$$

where $Q$ 's are arbitrary elements that make $V_{S 1}$ and $V_{S 2}$ unitary. Then, Eq. (A2) is changed to

$$
\frac{1}{\mathbb{N}}\left(|0\rangle \sum_{m=0}^{k} \sqrt{C_{m}}|m\rangle|x(0)\rangle+|1\rangle \sum_{n=1}^{k} \sqrt{D_{n}}|n-1\rangle|b\rangle\right) .
$$

The controlled operation $U_{c}=|0\rangle\left\langle 0\left|\otimes U_{0}+\right| 1\right\rangle\langle 1| \otimes$ $U_{1}+\cdots+|k\rangle\langle k| \otimes U_{k}$ is implemented afterwards, where $U_{k}=A^{k}$. The state of the whole system is

$$
\frac{1}{\mathbb{N}}|0\rangle \sum_{m=0}^{k} \sqrt{C_{m}}|m\rangle U_{m}|x(0)\rangle+\frac{1}{\mathbb{N}}|1\rangle \sum_{n=1}^{k} \sqrt{D_{n}}|n-1\rangle U_{n-1}|b\rangle .
$$



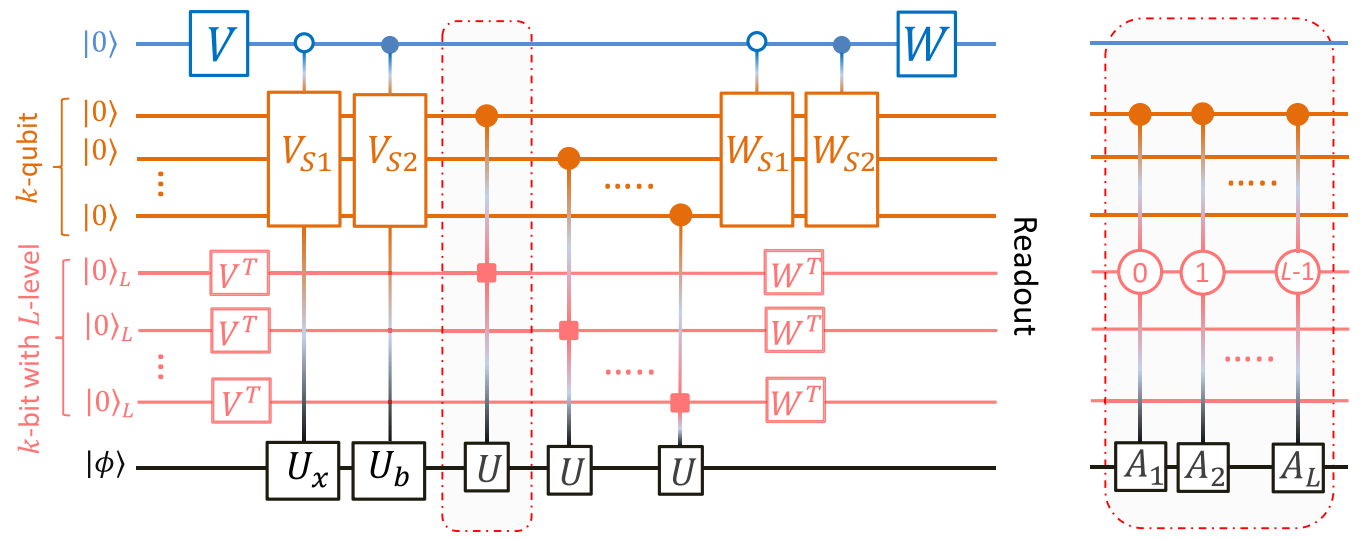

FIG. 6. Universal quantum circuit for solving any LDEs. The framework includes four parts: first ancilla register with one qubit, second ancilla register with $k$ qubits, third ancilla register with $k$ qudits where each qudit has $L$ levels, and a work system. All ancilla registers are initially prepared in the ground state $|0\rangle|0\rangle^{k}|0\rangle_{L}^{k}$. $|0\rangle_{L}$ denotes the ground state of an $L$-level system, which can be encoded by a $\log _{2}(L)$-qubit quantum system. Hence, all operations acting on the third ancilla register are $L \otimes L$ matrices. The red squares denote jointly controlled operations, with the corresponding circuit shown on the right. At the end of the circuit, we measure the state of the work system in the subspace where all ancilla registers are $|0\rangle|0\rangle^{k}|0\rangle_{L}^{k}$.

Subsequently, we implement the operations $W_{S 1}=V_{S 1}^{\dagger}$ and $W_{S 2}=V_{S 2}^{\dagger}$ controlled by the state of the first register on the second register, which leads to

$$
\frac{1}{\mathbb{N}}\left(|0\rangle|0\rangle^{\otimes T} \sum_{m=0}^{k} \frac{C_{m}}{\mathcal{C}} U_{m}|x(0)\rangle+|1\rangle|0\rangle^{\otimes T} \sum_{n=1}^{k} \frac{D_{n}}{\mathcal{D}} U_{n-1}|b\rangle\right)
$$

in the subspace where the second ancilla qubits are all $|0\rangle^{\otimes T}$. The last unitary operation $W=V^{\dagger}$ is applied on the first register. Analogously, we focus on the subspace where all ancilla qubits are $|0\rangle$, and the final state is

$$
\frac{1}{\mathbb{N}^{2}}|0\rangle|0\rangle^{\otimes T}\left(\sum_{m=0}^{k} C_{m} U_{m}|x(0)\rangle+\sum_{n=1}^{k} D_{n} U_{n-1}|b\rangle\right) .
$$

That is, if we measure the state of the work qubits in the subspace where the ancillas are $|0\rangle|0\rangle^{\otimes T}$, the result directly represents the solution to the LDE amplified by a factor $\mathbb{N}^{2}$. The successful probability of yielding the correct answer is

$$
\frac{1}{\left(\mathbb{N}^{2}\right)^{2}}\left(\sum_{m=0}^{k} C_{m}^{2}+\sum_{n=1}^{k} D_{n}^{2}\right) \approx \frac{1}{\mathbb{N}^{4}}
$$

\section{2. $A$ is nonunitary}

First, the nonunitary matrix $A$ can be decomposed into a linear combination of unitary operators $A=\sum_{i=1}^{L} \alpha_{i} A_{i}$, where the $A_{i}$ 's are unitary matrices. Thus, the solution can be written as

$$
\begin{aligned}
|x(t)\rangle \approx & \sum_{m=0}^{k} \frac{\|x(0)\|(\|\mathcal{M}\| t)^{m}\left(\sum_{i=1}^{L} \alpha_{i} A_{i}\right)^{m}}{m !}|x(0)\rangle \\
& +\sum_{n=1}^{k} \frac{\|b\|\|\mathcal{M}\|^{n-1} t^{n}\left(\sum_{i=1}^{L} \alpha_{i} A_{i}\right)^{n-1}}{n !}|b\rangle .
\end{aligned}
$$

To obtain the solution, we need to add the third ancilla register compared to the case when $A$ is unitary. The first ancilla register is still encoded in one qubit. The second ancilla register consists of $k$ qubits, and the third ancilla register consists of $k$ qudits where each qudit is an $L$-level quantum system.

A universal quantum circuit to solve any LDE is illustrated in Fig. 6. Initially, all ancilla registers are prepared in the ground state $|0\rangle|0\rangle^{k}|0\rangle_{L}^{k}$, where $|0\rangle_{L}$ denotes the ground state of an $L$-level quantum system. The work system employs the input state $|\phi\rangle$ to subsequently encode the vectors $|x(0)\rangle$ and $|b\rangle$. First, we implement the following operation $V$ on the first ancilla register:

$$
V=\left(\begin{array}{cc}
\frac{G_{1}}{\sqrt{G_{1}^{2}+G_{2}^{2}}} & \frac{G_{2}}{\sqrt{G_{1}^{2}+G_{2}^{2}}} \\
\frac{G_{2}^{2}}{\sqrt{G_{1}^{2}+G_{2}^{2}}} & -\frac{G_{1}}{\sqrt{G_{1}^{2}+G_{2}^{2}}}
\end{array}\right)
$$

where the parameters $G_{1}$ and $G_{2}$ are defined as

$$
\begin{gathered}
G_{1}=\sum_{m=0}^{k} \frac{\|x(0)\|(\|\mathcal{M}\| t)^{m}}{m !}\left(\sum_{i=1}^{L} \alpha_{i}\right)^{m}, \\
G_{2}=\sum_{n=1}^{k} \frac{\|b\|(\|\mathcal{M}\| t)^{n-1} t}{n !}\left(\sum_{i=1}^{L} \alpha_{i}\right)^{n-1} .
\end{gathered}
$$

In this way, we can encode the vectors $|x(0)\rangle$ and $|b\rangle$ by the controlled operations $U_{x}$ and $U_{b}$ on the work qubits, respectively. We then perform the controlled operations $V_{S 1}$ and $V_{S 2}$ on the second ancilla register depending on the state of the first ancilla register. $V_{S 1}$ and $V_{S 2}$ are $2^{k} \times 2^{k}$ matrices. The $m$ th element of the first column has the following definition:

$$
V_{S 1}^{(m, 1)}=\frac{v_{S 1}^{(m, 1)}}{\sqrt{\sum_{m}\left|v_{S 1}^{(m, 1)}\right|^{2}}}, \quad V_{S 2}^{(m, 1)}=\frac{v_{S 2}^{(m, 1)}}{\sqrt{\sum_{m}\left|v_{S 2}^{(m, 1)}\right|^{2}}},
$$

where

$$
\begin{aligned}
& v_{S 1}^{(m, 1)}=\left\{\begin{aligned}
\sqrt{\frac{\|x(0)\|(\|\| \| t)^{j}}{j !},} & m=2^{k}-2^{k-j}+1 \\
0, & \text { other case },
\end{aligned}\right. \\
& v_{S 2}^{(m, 1)}=\left\{\begin{aligned}
\sqrt{\frac{\|b\|(\|\mathcal{M}\| t)^{j-1} t}{j !},} & m=2^{k}-2^{k-j}+1 \\
0, & \text { other case. }
\end{aligned}\right.
\end{aligned}
$$


Besides, we apply the unitary operation $V_{T}$ on each $L$-level qudit of the third ancilla register, where $V_{T}$ is an $L \times L$ matrix. The $\ell$ th element of the first column in $V_{T}$ is

$$
V_{T}^{(\ell, 1)}=\frac{v_{T}^{(\ell, 1)}}{\sqrt{\sum_{\ell}\left|v_{T}^{(\ell, 1)}\right|^{2}}}, \quad \text { with } v_{T}^{(\ell, 1)}=\sqrt{\alpha_{i}},
$$

where $V_{\ell, 0}^{T}=\sqrt{\alpha_{i}}$. After implementing the unitary operations $V, V_{S 1}, V_{S 2}$, and $V_{T}$, the state of all ancilla registers will change from the initial state $|0\rangle|0\rangle^{k}|0\rangle_{L}^{k}$ to the following state:

$$
\begin{aligned}
& \frac{G_{1}}{\sqrt{G_{1}^{2}+G_{2}^{2}}}|0\rangle \sum_{m=0}^{2^{k}-1} V_{S 1}^{(m+1,0)}|m\rangle\left(\sum_{\ell=1}^{L} V_{T}^{(\ell, 1)}|\ell-1\rangle_{L}\right)^{\otimes k} \\
& +\frac{G_{2}}{\sqrt{G_{1}^{2}+G_{2}^{2}}}|1\rangle \sum_{m=0}^{2^{k}-1} V_{S 2}^{(m+1,0)}|m\rangle\left(\sum_{\ell=1}^{L} V_{T}^{(\ell, 1)}|\ell-1\rangle_{L}\right)^{\otimes k} .
\end{aligned}
$$

To entangle the ancilla and the work qubits, we perform the controlled operation $U$ on the work system, which is jointly controlled by the states of the second and third ancilla registers. If we focus on the subspace $|0\rangle|0\rangle^{k}|0\rangle_{L}^{k}$ of all ancilla registers, the state of the work system can be written as

$$
\begin{gathered}
\alpha|0\rangle \sum_{m} V_{S 1}^{(m+1,1)}|m\rangle\left(\sum_{\ell=1}^{L} V_{T}^{(\ell, 1)} A_{\ell}|\ell-1\rangle_{L}\right)^{\otimes j}|x(0)\rangle \\
+\beta|0\rangle \sum_{m} V_{S 2}^{(m+1,1)}|m\rangle\left(\sum_{\ell=1}^{L} V_{T}^{(\ell, 1)} A_{\ell}|\ell-1\rangle_{L}\right)^{\otimes j}|b\rangle .
\end{gathered}
$$

Here, the sum index $m=2^{k}-2^{k-j}, \alpha=G_{1} / \sqrt{G_{1}^{2}+G_{2}^{2}}$, and $\beta=G_{2} / \sqrt{G_{1}^{2}+G_{2}^{2}}$.

For decoding, we need to perform the inverse operations on all ancilla registers. The operations $W_{S 1}=V_{S 1}^{\dagger}$ and $W_{S 2}=$ $V_{S 2}^{\dagger}$ are implemented on the second register, which is controlled by the state of the first register, and we reverse the first and third registers by applying $W=V^{\dagger}$ and $W_{T}=V_{T}^{\dagger}$, respectively. Finally, we measure the state of work qubits in the subspace where all ancilla registers stay on the state $|0\rangle|0\rangle^{k}|0\rangle_{L}^{k} ;|0\rangle|0\rangle^{k}|0\rangle_{L}^{k}|\phi\rangle$ is changed to

$$
\begin{gathered}
\frac{1}{S}|0\rangle|0\rangle^{k}|0\rangle_{L}^{k}\left(\sum_{m=0}^{k} \frac{\|x(0)\|(\|\mathcal{M}\| A t)^{m}}{m !}\right)|x(0)\rangle \\
+\frac{1}{S}|0\rangle|0\rangle^{k}|0\rangle_{L}^{k}\left(\sum_{n=1}^{k} \frac{\|b\|(\|\mathcal{M}\| A)^{n-1} t^{n}}{n !}\right)|b\rangle,
\end{gathered}
$$

where $S=G_{1}+G_{2}$. One can obtain the solution to the LDE by multiplying $S$. If we directly measure the state of the work system at the end of circuit, the probability of successfully detecting the auxiliary state $|0\rangle|0\rangle^{k}|0\rangle_{L}^{k}$ is $P_{s}=\left\|A_{s}\right\|^{2} / S^{2}$, where $A_{s}$ is equal to

$$
\left.\sum_{j=0}^{k} \frac{\|x(0)\|(\|\mathcal{M}\| A t)^{j}}{j !}|x(0)\rangle+\sum_{j=1}^{k} \frac{\|b\|(\|\mathcal{M}\| A)^{j-1} t^{j}}{j !}|b\rangle\right) .
$$

$P_{S}$ is approximately $1 / S^{2} . S$ is the amplitude of the state of the work system on the subspace $|0\rangle|0\rangle^{k}|0\rangle_{L}^{k}$ of all registers. To improve the desired amplitude and obtain a near-100\% solution, we can adopt the robust obvious amplitude amplification by $S$ times before measurement.

\section{APPENDIX B: ERROR BOUNDS}

In this section, we analyze the infidelity between the exact solution $\tilde{x}(t)$ and the approximate solution $x(t)$, and give an upper bound of the error $\epsilon=\|x(t)-\tilde{x}(t)\|$. Since every square complex matrix is similar to a Jordan matrix, for an $n \times$ $n$ complex matrix $\mathcal{M}$, there exists an $n \times n$ invertible matrix $T$ such that $\mathcal{M}=T J T^{-1}$, where $J=J_{1} \oplus J_{2} \oplus \cdots \oplus J_{m}$, and $J_{i}$ is a $d_{i} \times d_{i}$ Jordan block with eigenvalues $\lambda_{i}$,

$$
J_{i}=\left(\begin{array}{cccccc}
\lambda_{i} & 1 & 0 & \cdots & 0 & 0 \\
0 & \lambda_{i} & 1 & \cdots & 0 & 0 \\
0 & 0 & \lambda_{i} & \cdots & 0 & 0 \\
\cdots & \cdots & \cdots & \cdots & \cdots & \ldots \\
0 & 0 & 0 & \cdots & \lambda_{i} & 1 \\
0 & 0 & 0 & \cdots & 0 & \lambda_{i}
\end{array}\right),
$$

for $i=1,2, \ldots m$, and $\sum_{i=1}^{m} d_{i}=n$. Thus, $e^{\mathcal{M} t}=T e^{J t} T^{-1}$. One can compute that

$$
e^{J t}=\oplus_{i=1}^{m} e^{\lambda_{i} t} J_{i}^{\prime}
$$

where

$$
J_{i}^{\prime}=\left(\begin{array}{cccccc}
1 & t & \frac{1}{2} t^{2} & \ldots & \frac{1}{\left(d_{i}-2\right) !} t^{d_{i}-2} & \frac{1}{\left(d_{i}-1\right) !} t^{d_{i}-1} \\
0 & 1 & t & \ldots & \frac{1}{\left(d_{i}-3\right) !} t^{d_{i}-3} & \frac{1}{\left(d_{i}-2\right) !} t^{d_{i}-2} \\
0 & 0 & 1 & \ldots & \frac{1}{\left(d_{i}-4\right) !} t^{d_{i}-4} & \frac{1}{\left(d_{i}-3\right) !} t^{d_{i}-3} \\
\ldots & \ldots & \ldots & \ldots & \cdots & \cdots \\
0 & 0 & 0 & \ldots & 1 & t \\
0 & 0 & 0 & \ldots & 0 & 1
\end{array}\right)
$$

is a $d_{i} \times d_{i}$ complex matrix. It follows that

$$
\left\|e^{J t}\right\|=\max \left\{e^{t \operatorname{Re} \lambda_{i}}\left\|J_{i}^{\prime}\right\| \mid i=1,2, \ldots m\right\},
$$

where $\left\|J_{i}^{\prime}\right\|$ denotes the spectral norm, that is, the largest singular value of $J_{i}^{\prime}$. A Taylor expansion of $e^{z}$ with Lagrange remainder reads

$$
e^{z}=\sum_{i=1}^{k} \frac{z^{k}}{k !}+\frac{e^{\theta z}}{(k+1) !} z^{k+1}
$$

where $0<\theta<1$ is a constant. Let

$$
\begin{aligned}
C= & \left(\|x(0)\|+\frac{\|b\|}{\|\mathcal{M}\|}\right)\|T\|\left\|T^{-1}\right\| \\
& \times \max \left\{e^{t \operatorname{Re}_{i}}\left\|J_{i}^{\prime}\right\| \mid i=1,2, \ldots, m\right\} .
\end{aligned}
$$

Then, the error is given by

$$
\epsilon=\|x(t)-\tilde{x}(t)\| \leqslant \frac{\|\mathcal{M} t\|^{k+1}}{(k+1) !} C .
$$

When $k$ is sufficiently large,

$$
(k+1) ! \approx \sqrt{2(k+1) \pi}\left(\frac{k+1}{e}\right)^{k+1},
$$

and it follows that

$$
\frac{\sqrt{2 \pi}}{C} \epsilon \leqslant\left(\frac{e\|\mathcal{M} t\|}{k+1}\right)^{k+1} \frac{1}{\sqrt{k+1}},
$$


and hence,

$$
\ln \frac{\sqrt{2 \pi}}{C} \epsilon \leqslant(k+1)[\ln \|e \mathcal{M} t\|-\ln (k+1)]-\frac{1}{2} \ln (k+1)
$$

SO

$$
\ln \frac{\sqrt{2 \pi}}{C} \epsilon \leqslant(k+1)[\ln \|e \mathcal{M} t\|-\ln (k+1)] .
$$

Since

$$
\ln (k+1)-\ln \|e \mathcal{M} t\| \geqslant \frac{k+1-\|e \mathcal{M} t\|}{k+1},
$$

we have

$$
k+1 \leqslant\|e \mathcal{M} t\|+\ln \frac{C}{\sqrt{2 \pi}} \frac{1}{\epsilon} .
$$

Therefore, $k \leqslant \ln \frac{e^{\|e \mathcal{M} t\|-1} C}{\sqrt{2 \pi}} \frac{1}{\epsilon}$. Let $C_{0}=\frac{e^{\|e \mathcal{M} t\|-1} C}{\sqrt{2 \pi}}$. Then, $k \leqslant$ $\ln \frac{C_{0}}{\epsilon}$, which implies that the larger the value of $k$, the smaller the error $\epsilon$.

\section{APPENDIX C: THE COMPLEXITY ANALYSIS FOR NONUNITARY $A$}

Generally speaking, the cost of a quantum algorithm involves two aspects. One aspect is qubit resource corresponding to space complexity. The other aspect is gate complexity corresponding to time complexity. We have given the complexity analysis for unitary $A$. In this section, we present a detailed discussion about complexity for our algorithm when $A$ is nonunitary.

Qubit resources. The number of total ancillary qubits is $1+k+k \log L$, where $L$ is the number of unitary operators $A_{i}$ when $A$ is decomposed into $A=\sum_{i=1}^{L} \alpha_{i} A_{i}$. The qubit number of the work system is $\log N$. Hence, the total qubit resource in our algorithm is $\log N+k \log L+k+1$.

Gate complexity. The analysis is the same as that for the case of unitary $A$ in the main text. In the encoding part, we need $O(\log 2 N)$ steps to prepare the state $C|0\rangle|x(0)\rangle+D|1\rangle|b\rangle$. The controlled operators $V_{S 1}$ and $V_{S 2}$ can be regarded as two general $2^{k+1} \times 2^{k+1}$ unitary matrices. It can be decomposed into $O\left((k+1)^{3} 4^{k+1}\right)$ elementary gates via QR decomposition [33]. Each operation $V^{T}$ can be decomposed into $O\left((\log L)^{3} L^{2}\right) \sim O\left(L^{2}\right)$ elementary gates. Hence, we need $O\left(\log 2 N+(k+1)^{3} 4^{k+1}+k(\log L)^{3} L^{2}\right) \sim$ $O\left(\log N+k^{3} 4^{k}+k L^{2}\right)$ steps to realize the encoding part.

In the middle part, the controlled operations $U$ can be decomposed into $O(k L(\log L+1) \log N) \sim O(k L \log L \log N)$ basic gates [34]. The decoding part has the same gate complexity compared to the encoding. So for nonunitary $A$, our algorithm requires $O\left(k L \log L \log N+k^{3} 4^{k}+k L^{2}\right)$ steps to obtain the solution.

From the above analysis, we see that the gate complexity mainly relies on the preparation of $\left|\psi_{1}\right\rangle$. Actually, the state $\left|\psi_{1}\right\rangle$ can be prepared by using the qRAM method [30]. qRAM claims the gate complexity for preparing an arbitrary $P$ dimensional state is $O(\log P)$ after the quantum memory cell is established. Hence, we can also analyze the gate complexity for the qRAM method.

Unitary A. The dimensions of the work system, first ancillary register, and second ancillary register are $N, k+1$, and 2, respectively. The dimension of the whole system is $2 N(k+1)$. Thus, the gate complexity for preparing state $\left|\psi_{1}\right\rangle$ is $O(\log (2 N(k+1)))$. The total gate complexity will be reduced to $O(k \log k \log N+\log k N) \sim O(k \log k \log N)$.

Nonunitary A. The dimensions of the work system, first ancillary register, second ancillary register, and third ancillary register are $N, 2,2^{k}$, and $k L$, respectively. The dimension of the whole system is $N 2^{k+1} k L$. The gate complexity to prepare $\left|\psi_{1}\right\rangle$ is $O\left(\log \left(N 2^{k+1} k L\right)\right)$. The total gate complexity will be reduced to $O(k L \log L \log N+$ $\left.\log \left(N 2^{k+1} k L\right)\right)=O(k L \log L \log N+\log N+(k+1)+$ $\log k+L) \sim O(k L \log L \log N)$.

We summarize the qubit resource and gate complexity of our algorithm in Table II. Clearly, the performance of our algorithm depends on both $k$ and $L$. If $4^{k}$ or $L$ is polynomial in $N$, our algorithm may not outperform classical algorithms (polynomial speedup is still possible). This is very similar during the analysis of complexity in the HHL algorithm. For the situations when $4^{k}$ and $L$ are both polylogarithmic in $N$, our algorithm achieves an exponential speedup. Recall that the Taylor order $k$ determines the precision of the solution by $k=\ln \left(C_{0} / \epsilon\right)$; that is, $k$ improves the precision exponentially. Therefore, in most cases, a fairly small $k$ is sufficient for approximating a solution with remarkable precision, and we can omit its contribution to the complexity.

In summary, in most (at least certain) cases, we only need to consider the system size $N$ when analyzing the complexity of our algorithm. The complexity scales in $O(\log N)$.

Next we discuss the success probability of solving LDEs with our algorithms. The solution of an LDE after the $k$-order Taylor expansion can be written as

$$
x(t) \approx \sum_{m=0}^{k} \frac{(\mathcal{M} t)^{m}}{m !} x(0)+\sum_{n=1}^{k} \frac{\mathcal{M}^{n-1} t^{n}}{n !} b .
$$

Now let us define a constant number $G$,

$$
\begin{aligned}
G= & \max \left\{\|x(0)\|(\|\mathcal{M}\| t)^{k},\|b\|(\|\mathcal{M}\| t)^{k}\right\}, \\
& \text { if }\|\mathcal{M}\| t>1,\|\mathcal{M}\|>1, \\
G= & \max \left\{\|x(0)\|(\|\mathcal{M}\| t)^{k} t,\|b\|(\|\mathcal{M}\| t)^{k} t\right\}, \\
& \text { if }\|\mathcal{M}\| t>1,\|\mathcal{M}\|<1, \\
G= & \max \{\|x(0)\|,\|b\|\}, \quad \text { if }\|\mathcal{M}\| t \leqslant 1,\|\mathcal{M}\|>1, \\
G= & \max \{\|x(0)\| /\|\mathcal{M}\|,\|b\| /\|\mathcal{M}\|\}, \\
& \text { if }\|\mathcal{M}\| t \leqslant 1,\|\mathcal{M}\|<1 .
\end{aligned}
$$

We divide both sides of the equation by this $G$. The new solution, $x^{\prime}(t)=x(t) / G$, is the same as the original solution $x(t)$ up to a constant. In terms of the quantum state, $x^{\prime}(t)$ has the form

$$
\begin{aligned}
\left|x^{\prime}(t)\right\rangle & \approx \frac{1}{\mathbb{N}^{2}}\left(\sum_{m=0}^{k} \frac{\|x(0)\|(\|\mathcal{M}\| A t)^{m}}{G m !}|x(0)\rangle\right. \\
& \left.+\sum_{n=1}^{k} \frac{\|b\|(\|\mathcal{M}\| A)^{n-1} t^{n}}{G n !}|b\rangle\right),
\end{aligned}
$$


TABLE II. Required qubit resources and gate complexity in our algorithm. $N$ and $k$ are the dimensions of the work system and Taylor order, respectively. $L$ is the number of unitary operations $A_{i}$ when we decompose nonunitary $A$ into $A=\sum_{i=1}^{L} \alpha_{i} A_{i}$. It shows that our algorithm exhibits an exponential speedup over its classical counterpart in certain circumstances.

\begin{tabular}{lcc}
\hline \hline Case & Unitary $A$ & Nonunitary A \\
\hline Qubit & $1+\log (k+1)+\log N$ & $\log N+k \log L+k+1$ \\
Gate & $O\left(k \log k \log N+\log N+k^{2}\right)$ & $O\left(k L \log L \log N+k^{3} 4^{k}+k L^{2}\right)$ \\
Gate (qRAM) & $O(k \log k \log N)$ & $O(k L \log L \log N)$ \\
\hline \hline
\end{tabular}

where $\quad \mathbb{N}^{2}=\sum_{m=0}^{k} C_{m}+\sum_{n=1}^{k} D_{n} \quad$ with $\quad C_{m}=$ $\|x(0)\|(\|\mathcal{M}\| t)^{m} / G m ! \quad$ and $\quad D_{n}=\|b\|(\|\mathcal{M}\|)^{n-1} t^{n} / G n !$. Hence, the success probability to obtain the solution is

$$
\begin{aligned}
P= & \frac{1}{\mathbb{N}^{4}} \frac{1}{G^{2}} \| \sum_{m=0}^{k} \frac{\|x(0)\|(\|\mathcal{M}\| A t)^{m}}{m !}|x(0)\rangle \\
& +\sum_{n=1}^{k} \frac{\|b\|(\|\mathcal{M}\| A)^{n-1} t^{n}}{n !}|b\rangle \|^{2},
\end{aligned}
$$

where $G$ is the constant number defined by Eq. (C2).

(i) For the term $\frac{1}{\mathbb{N}^{4}}$, we know

$$
\begin{aligned}
\mathbb{N}^{2} & =\sum_{m=0}^{k} C_{m}+\sum_{n=1}^{k} D_{n} \\
& =\sum_{m=0}^{k} \frac{\|x(0)\|(\|\mathcal{M}\| t)^{m}}{G m !}+\sum_{n=1}^{k} \frac{\|b\|(\|\mathcal{M}\|)^{n-1} t^{n}}{G n !} \\
& \leqslant \sum_{m=0}^{k} \frac{1}{m !}+\sum_{n=1}^{k} \frac{1}{n !} \leqslant e+e-1=2 e-1 .
\end{aligned}
$$

Hence,

$$
\frac{1}{\mathbb{N}^{4}} \geqslant \frac{1}{(2 e-1)^{2}} .
$$

(ii) Now consider the term

$$
\begin{aligned}
S= & \| \sum_{m=0}^{k} \frac{\|x(0)\|(\|\mathcal{M}\| A t)^{m}}{m !}|x(0)\rangle \\
& +\sum_{n=1}^{k} \frac{\|b\|(\|\mathcal{M}\| A)^{n-1} t^{n}}{n !}|b\rangle \|^{2} \\
\approx & \| e^{\|\mathcal{M}\| A t}|x(0)\rangle\left\|x_{0}\right\|+\|b\|\left(e^{\|\mathcal{M}\| A t}-I\right)(\|\mathcal{M}\| A)^{-1}|b\rangle \|^{2} .
\end{aligned}
$$

First, let us assume the $\mathcal{M}$ is anti-Hermitian. Then

$$
\begin{aligned}
S= & \|x(0)\|^{2}+2\|b\| \cdot\left\|x_{0}\right\|\left\langle b\left|\left(e^{\|\mathcal{M}\| A t}-I\right)(\|\mathcal{M}\| A)^{-1}\right| x(0)\right\rangle \\
& +\|b\|^{2} \|\left(e^{\|\mathcal{M}\| A t}-I\right)(\|\mathcal{M}\| A)^{-1}|b\rangle \|^{2} . \\
\geqslant & \|x(0)\|^{2}-2 \frac{\|b\| \cdot\left\|x_{0}\right\|}{\|\mathcal{M}\|} \|\left(e^{\|\mathcal{M}\| A t}-I\right) A^{-1}|b\rangle \| \\
& +\frac{\|b\|^{2}}{\|\mathcal{M}\|^{2}} \|\left(e^{\|\mathcal{M}\| A t}-I\right) A^{-1}|b\rangle \|^{2} .
\end{aligned}
$$

When $\|x(0)\| \leqslant \frac{\|b\|}{2\|\mathcal{M}\|} \|\left(e^{\|\mathcal{M}\| A t}-I\right) A^{-1}|b\rangle \|$, we can get $S \geqslant$ $\|x(0)\|^{2}$. It means the success probability of our algorithm has the lower boundary,

$$
P \geqslant \frac{\|x(0)\|^{2}}{(2 e-1)^{2} G^{2}} .
$$

If $\mathcal{M}$ is not anti-Hermitian, we can reconstruct an antiHermitian operator $\widetilde{\mathcal{M}}$,

$$
\widetilde{\mathcal{M}}=\left(\begin{array}{cc}
0 & \mathcal{M} \\
-\mathcal{M}^{\dagger} & 0
\end{array}\right),
$$

and then solve the LDE. Analysis of the success probability is the same as that of the anti-Hermitian $\mathcal{M}$ case. Hence, there is no success probability problem in our algorithm as long as $\|x(0)\| \leqslant \frac{\|b\|}{2\|\mathcal{M}\|} \|\left(e^{\|\mathcal{M}\| A t}-I\right) A^{-1}|b\rangle \|$.

On the other hand, it does not mean that the success probability of our algorithm must be exponentially small if $\|x(0)\| \geqslant \frac{\|b\|}{2\|\mathcal{M}\|} \|\left(e^{\|\mathcal{M}\| A t}-I\right) A^{-1}|b\rangle \|$. For instance, when $b=$ $0, P$ is reduced to $\frac{\|x(0)\|^{2}}{N^{4} G^{2}}$, which is also independent of system size.

\section{APPENDIX D: AN ALTERNATIVE APPROACH OF OUR ALGORITHM}

When the matrix $A$ is nonunitary, we provide an alternative approach to solve LDEs. The solution can be written as

$$
\begin{aligned}
x(t) \approx & \sum_{m=0}^{k} \frac{\|x(0)\|(\|\mathcal{M}\| A t)^{m}}{m !}|x(0)\rangle \\
& +\sum_{n=1}^{k} \frac{\|b\|(\|\mathcal{M}\| A)^{n-1} t^{n}}{n !}|b\rangle,
\end{aligned}
$$

where $A$ is a normalized matrix satisfying $\|A\| \leqslant 1$. Then, $A=$ $B+i C$ where $B$ and $C$ are the real and imaginary parts with

$$
B=\frac{1}{2}\left(A+A^{\dagger}\right), C=\frac{1}{2 i}\left(A-A^{\dagger}\right) .
$$

It is known that any real matrix can be decomposed into the linear combination of two unitary matrices. Hence,

$$
B=1 / 2\left(F_{1}+F_{2}\right), C=1 / 2\left(F_{3}+F_{4}\right),
$$

where the matrices $F_{1}, F_{2}, F_{3}$, and $F_{4}$ are all unitary. Their definitions are

$$
\begin{aligned}
& F_{1}=B+i \sqrt{I-B^{2}}, F_{2}=B-i \sqrt{I-B^{2}}, \\
& F_{3}=C+i \sqrt{I-C^{2}}, F_{4}=C-i \sqrt{I-C^{2}},
\end{aligned}
$$

respectively. Then, we can obtain the relationship between the matrices $A$ and $F_{i}$ 's:

$$
A=\frac{1}{2}\left(F_{1}+F_{2}\right)+\frac{i}{2}\left(F_{3}+F_{4}\right) .
$$


If the coefficient $i$ is absorbed into $F_{3}$ and $F_{4}$,

$$
F_{3}=i C-\sqrt{I-C^{2}}, F_{4}=i C+\sqrt{I-C^{2}},
$$

we have

$$
A=\frac{1}{2}\left(F_{1}+F_{2}+F_{3}+F_{4}\right) .
$$

The former is a linear combination of only four unitary matrices. In this situation, the solution $x(t)$ can be written as

$$
\begin{aligned}
x(t) \approx & \sum_{m=0}^{k} \frac{\|x(0)\|\left(\sum_{i=1}^{4} \frac{\|\mathcal{M}\|}{2} F_{i} t\right)^{m}}{m !}|x(0)\rangle \\
& +\sum_{n=1}^{k} \frac{\|b\|\left(\sum_{i=1}^{4} \frac{\|\mathcal{M}\|}{2} F_{i}\right)^{n-1} t^{n}}{n !}|b\rangle .
\end{aligned}
$$

It shows that the number of unitary operators acting on $|x(0)\rangle$ is less than $\frac{4^{k+1}-1}{3}$ and the number of unitary operators acting on $|b\rangle$ is less than $\frac{4^{k}-1}{3}$. Thus, the number of required ancilla qubits is about $\log _{2}\left(\frac{4^{k+1}-1}{3}\right)+1 \approx 2 k$. The solution $|x(t)\rangle$ can be further expressed as

$$
x(t) \approx \sum_{m=1}^{\frac{4^{k+1}-1}{3}} C_{m} U_{m}|x(0)\rangle+\sum_{n=1}^{\frac{4^{k}-1}{3}} D_{n} U_{n}|b\rangle .
$$

The parameters $C_{m}$ and $D_{n}$ satisfy

$$
\begin{gathered}
C_{m}=\left\{\begin{array}{l}
\|x(0)\|, \mathrm{m}=1 \\
\frac{\|x(0)\|(\|\mathcal{M}\| t / 2)^{j}}{j !} \log _{4}(m-1) \leqslant j, j \in 1,2, \ldots, k,
\end{array}\right. \\
D_{n}= \begin{cases}\|b\| t, & \mathrm{n}=1 \\
\frac{\|b\|(\|\mathcal{M}\| t / 2)^{j} t}{j !} & \log _{4}(n-1) \leqslant j, j \in 1,2, \ldots, k-1 .\end{cases}
\end{gathered}
$$

Similarly, by defining $\mathcal{C}=\sqrt{\sum C_{m}}$ and $\mathcal{D}=\sqrt{\sum D_{n}}$, we obtain $|x(t)\rangle$ :

$$
\mathcal{C}^{2} \frac{\left(\sum_{m=1}^{\frac{4^{k+1}-1}{3}} C_{m} U_{m}\right)}{\mathcal{C}^{2}}|x(0)\rangle+\mathcal{D}^{2} \frac{\left(\sum_{n=1}^{\frac{4^{k}-1}{3}} D_{n} U_{n}\right)}{\mathcal{D}^{2}}|b\rangle .
$$

This alternative approach provides a new way to realize the solution of any type of LDE.

\section{APPENDIX E: EXPERIMENTAL PROTOCOL}

In experiment, the parameters of the target LDE are chosen as follows. $\mathcal{M}$ is chosen as $\mathcal{M}=I \otimes I+2 I \otimes \sigma_{x}$. Starting from the initial state $|\phi\rangle$, we encode the vector $|x(0)\rangle$ by applying a two-qubit operation $U_{x}$ on $|\phi\rangle$, and the offset vector $|b\rangle$ by applying an additional rotation $U_{b}$ on $|\phi\rangle$. More specifically,

$$
|\phi\rangle=R_{y}^{\mathrm{A}}(\beta) R_{y}^{\mathrm{B}}(\beta)|00\rangle,|x(0)\rangle=U_{x}|\phi\rangle,|b\rangle=U_{b}|\phi\rangle,
$$

where $R_{y}^{j}(\beta)$ denotes a local rotation $R_{y}^{j}(\beta)=e^{-i \beta \sigma_{y}^{j} / 2}$ acting on qubit $j$ with angle $\beta$ about the $y$ axis. The order $k$ in the Taylor expansion directly determines the accuracy of the approximate solution $x(t)$. As shown in Fig. 3, we present a detailed quantum circuit with four qubits for realizing the solution $x(t) . \mathcal{C}$ and $\mathcal{D}$ in operations $V$ and $W$ are defined as

$$
\mathcal{C}=\sqrt{C_{1}+C_{2}}, \quad \mathcal{D}=\sqrt{D_{1}+D_{2}} .
$$

Operations $V_{S 1}$ and $V_{S 2}$ are chosen as

$$
V_{S 1}=\frac{1}{\mathcal{C}}\left(\begin{array}{ll}
\sqrt{C_{1}} & N \\
\sqrt{C_{2}} & N
\end{array}\right), \quad V_{S 2}=\frac{1}{\mathcal{D}}\left(\begin{array}{ll}
\sqrt{D_{1}} & N \\
\sqrt{D_{2}} & N
\end{array}\right) .
$$

Here,

$$
\begin{aligned}
& C_{1}=1+t+\frac{5 t^{2}}{2}+\frac{13 t^{3}}{6}+\frac{41 t^{4}}{24}, \\
& C_{2}=2 t+2 t^{2}+\frac{7 t^{3}}{3}+\frac{5 t^{4}}{3}, \\
& D_{1}=t+\frac{t^{2}}{2}+\frac{5 t^{3}}{6}+\frac{13 t^{4}}{24}, \quad D_{2}=t^{2}+\frac{2 t^{3}}{3}+\frac{7 t^{4}}{12} .
\end{aligned}
$$

$N$ 's are arbitrary elements that make $V_{S 1}$ and $V_{S 2}$ unitary, which can be determined using the Gram-Schmidt method. The other operations are $W_{S 1}=V_{S 1}^{\dagger}$ and $W_{S 2}=V_{S 2}^{\dagger}$. The controlled operation $U_{c}$ is simplified to the controlled-NOT operation $U_{c}=I \otimes\left(|0\rangle\langle 0|\otimes I+| 1\rangle\langle 1| \otimes \sigma_{x}\right) \otimes I$.

\section{APPENDIX F: QUANTUM STATE TOMOGRAPHY}

In NMR, the measurement is realized by detecting the freeinduction decay (FID) signal. When the spins in the detecting coil remove to the thermal equilibrium, the electric current in the coil will create and can be detected by the NMR detector and a time-domain FID signal is induced. We perform Fourier transformation on the FID signal to extract the information of spins including the oscillating frequencies and the amplitudes and phases of the transverse magnetization. Hence, we can process the experimental spectra such as the fitting to measure the values of the observables. The NMR platform can only measure the expectations of the single-coherent operators; for example, the observed qubit is the transverse component $\sigma_{x}$ or $\sigma_{y}$ and the remaining qubits are all $\sigma_{z}$ or $I$ with Pauli matrices $\sigma_{x}, \sigma_{y}$, and $\sigma_{z}$. It means that we can also measure other operators by some designed readout pulses before the FID signal acquisition and then realize quantum state tomography (QST). For a four-qubit quantum state $\rho$, it can be decomposed in the complete Pauli basis,

$$
\rho=\sum_{i, j, k, l=0}^{3} \mu_{i j k l} \sigma_{i} \otimes \sigma_{j} \otimes \sigma_{k} \otimes \sigma_{l},
$$

$\sigma_{0}=I, \sigma_{1}=\sigma_{x}, \sigma_{2}=\sigma_{y}, \sigma_{3}=\sigma_{z} \cdot \mu_{i j k l}$ is the expectation of the operator $\sigma_{i} \sigma_{j} \sigma_{k} \sigma_{l}$. It is $\mu_{i j k l}=\operatorname{Tr}\left(\rho \sigma_{i} \sigma_{j} \sigma_{k} \sigma_{l}\right)$. QST is a way to determine all the unknown coefficients $\mu_{i j k l}$ in the state $\rho$. Except for single-coherent operators like $\sigma_{x} \sigma_{z} \sigma_{z} \sigma_{z}$, we can also measure the high-coherent operators with the assistance of the readout pulses which are $\pi / 2$ rotations on the qubits. For instance, we need a rotation $\exp \left(-i \pi / 4 \sigma_{x}^{2}-\right.$ $\left.i \pi / 4 \sigma_{x}^{3}-i \pi / 4 \sigma_{x}^{4}\right)$ on nonobserved qubits to transfer a desired operator $\sigma_{x} \sigma_{y} \sigma_{y} \sigma_{y}$ to the detectable single-coherent operator $\sigma_{x} \sigma_{z} \sigma_{z} \sigma_{z}$. Obviously, we can design some readout pulses to measure all the coefficients $\mu_{i j k l}$ in the QST.

In experiments, these readout pulses are as well realized by the optimized shaped pulses with a numerical fidelity of about $99.8 \%$. As a result, the final density matrices of four repeated experiments for every $\beta$ are reconstructed as $\rho_{\text {expt }}$ and the average fidelity $94.64 \%$ is obtained. Figure 7 presents the comparison between the reconstructed and 


\section{$\operatorname{Re}\left(\rho_{\text {expt }}\right)$ and $\operatorname{Im}\left(\rho_{\text {expt }}\right)$}
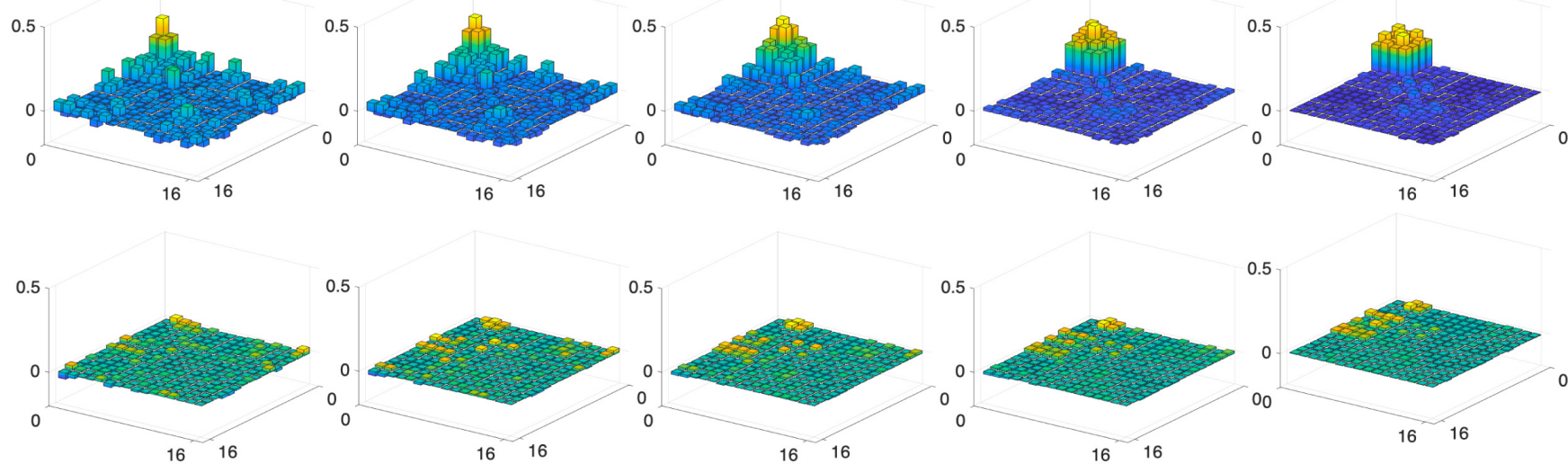

\section{$\operatorname{Re}\left(\rho_{t h}\right)$ and $\operatorname{Im}\left(\rho_{t h}\right)$}
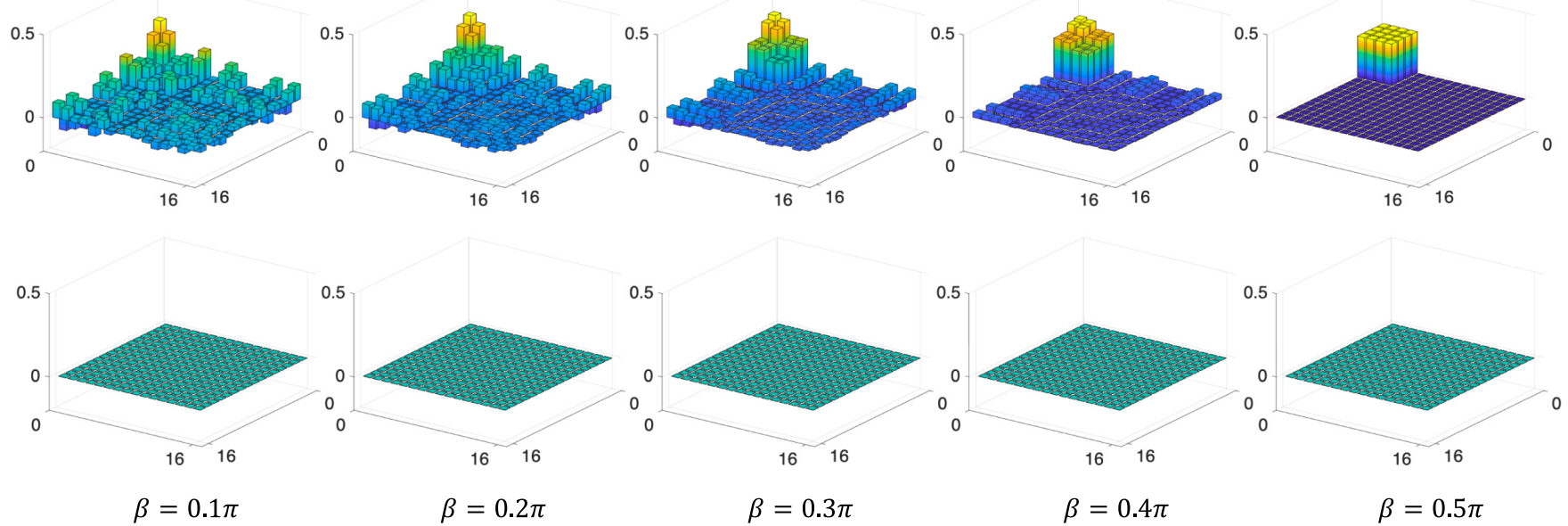

FIG. 7. The real and imaginary parts of the density matrices $\rho_{\text {expt }}$ and $\rho_{t h}$ for five $\beta$ 's. The first and second rows present the real and imaginary parts of the reconstructed density matrices $\rho_{\text {expt }}$, respectively. The third and fourth rows show the the real and imaginary parts of the ideal density matrices $\rho_{t h}$. The average fidelity between $\rho_{\text {expt }}$ and $\rho_{t h}$ for all $\beta$ 's is about $94.64 \%$.

expected density matrices for every $\beta$. The experimental results have a great agreement with the theoretical density matrices and reveal the quantum circuit is precisely implemented in experiments.

\section{APPENDIX G: ERROR ANALYSIS IN EXPERIMENTS}

The infidelity of the final density matrix $\rho_{\text {expt }}$ (about $5.36 \%$ ) is attributed to the imperfections of the initial state preparation, the imprecisions and inhomogeneity of the optimized pulses, the decoherence effect, and the readout error in tomography. First, there is about $1.07 \%$ error related to the infidelity of the initial state preparation after state tomography. Second, the shaped pulses have distortions in practice as the pulse generator has about 5\% fluctuation in amplitude. Our simulation gives on average $0.84 \%$ error to the infidelity of $\rho_{\text {expt }}$.

The decoherence effect is usually the main error source in current quantum computation. It always makes the dynamical evolution deviate from the ideal dynamics. To evaluate the influence of decoherence on our experimental results, we first suppose a simple decoherence model where each qubit undergoes an individual relaxation channel after each slice in the shaped pulses, and then we simulate the dynamics of the

TABLE III. Numerical estimation of errors. For every value of $\beta$, we simulate the infidelities $\bar{F}_{\sigma}^{\text {PPS }}, \bar{F}_{\sigma}^{\text {inhomo }}, \bar{F}_{\sigma}^{\text {decoh }}$, and $\bar{F}_{\sigma}^{\text {tomo }}$ caused by the imperfections of the PPS preparation, the inhomogeneity of the optimized pulses, the decoherence effect, and the error in state tomography, respectively.

\begin{tabular}{lccccc}
\hline \hline$\beta$ & $0.1 \pi$ & $0.2 \pi$ & $0.3 \pi$ & $0.4 \pi$ & $0.5 \pi$ \\
\hline $\bar{F}_{\sigma}^{\text {PPS }}$ & $1.070 \%$ & $1.070 \%$ & $1.070 \%$ & $1.070 \%$ & $1.070 \%$ \\
$\bar{F}_{\sigma}^{\text {inhomo }}$ & $0.859 \%$ & $0.933 \%$ & $0.948 \%$ & $0.864 \%$ & $0.604 \%$ \\
$\bar{F}_{\sigma}^{\text {deco }}$ & $1.272 \%$ & $1.368 \%$ & $1.258 \%$ & $1.163 \%$ & $0.869 \%$ \\
$\bar{F}_{\sigma}^{\text {tomo }}$ & $1.008 \%$ & $0.960 \%$ & $1.056 \%$ & $1.277 \%$ & $1.841 \%$ \\
$\bar{F}_{\sigma}^{\text {total }}$ & $4.209 \%$ & $4.331 \%$ & $4.332 \%$ & $4.374 \%$ & $4.384 \%$ \\
\hline \hline
\end{tabular}


shaped pulses including the decoherence effect for our qubits. In detail, the time propagator including decoherence model can be described as

$$
\mathcal{C}=\epsilon \cdot \mathcal{C}_{M} \cdot \epsilon \cdot \mathcal{C}_{M-1} \cdots \epsilon \cdot \mathcal{C}_{m} \cdots \epsilon \cdot \mathcal{C}_{2} \cdot \epsilon \cdot \mathcal{C}_{1},
$$

where $\mathcal{C}_{m}$ acting on the density matrix $\rho$ means $\mathcal{C}_{m}(\rho)=$ $\mathrm{e}^{-i H_{m} \tau} \rho \mathrm{e}^{i H_{m} \tau} . H_{m}$ is the total Hamiltonian of the $m$ th slice and $\tau$ is the duration of each slice. $\epsilon$ is the combination of the relaxation channel for each qubit. When a density matrix $\rho$ is fed into such a channel, $\rho$ is changed to

$$
\rho \rightarrow \epsilon(\rho)=\epsilon_{4} \circ \epsilon_{3} \circ \epsilon_{2} \circ \epsilon_{1}(\rho) .
$$

Here, $\epsilon_{i}$ means the amplitude damping and phase damping channel of the $i$ th qubit. According to the above model, the error caused by the decoherence effect is about $1.19 \%$.

Another potential error source is the measurement error. This is usually combined with the spectra fitting inaccuracy and is difficult to address. We estimate it by adding a fluctuation on the measured values of the coefficients (Pauli basis). This error is simulated to be about $1.23 \%$ on average.

Assuming all errors are additive, the total error estimation gives on average $4.33 \%$ (see Table III), which is about $1 \%$ smaller than the infidelity of $\rho_{\text {expt }}$. As the difference is quite small, our error estimation is consistent with the experimental results.
[1] D. Kothe et al., Science at the petascale 2009: Pioneering applications point the way, National Center for Computational Sciences (2009), http://www.olcf.ornl.gov/wp-content/uploads/ 2010/03/PetaDocLowRes2-9-10.pdf (accessed 30 September, 2013).

[2] P. W. Shor, SIAM Rev. 41, 303 (1999).

[3] D. R. Simon, SIAM J. Comput. 26, 1474 (1997).

[4] L. K. Grover, Phys. Rev. Lett. 79, 4709 (1997).

[5] A. W. Harrow, A. Hassidim, and S. Lloyd, Phys. Rev. Lett. 103, 150502 (2009).

[6] Y. Subaş1, R. D. Somma, and D. Orsucci, Phys. Rev. Lett. 122, 060504 (2019).

[7] S. Barz, I. Kassal, M. Ringbauer, Y. O. Lipp, B. Dakić, A. Aspuru-Guzik, and P. Walther, Sci. Rep. 4, 6115 (2014).

[8] X.-D. Cai, C. Weedbrook, Z.-E. Su, M.-C. Chen, M. Gu, M.-J. Zhu, L. Li, N.-L. Liu, C.-Y. Lu, and J.-W. Pan, Phys. Rev. Lett. 110, 230501 (2013).

[9] J. Pan, Y. Cao, X. Yao, Z. Li, C. Ju, H. Chen, X. Peng, S. Kais, and J. Du, Phys. Rev. A 89, 022313 (2014).

[10] J. Wen, X. Kong, S. Wei, B. Wang, T. Xin, and G. Long, Phys. Rev. A 99, 012320 (2019).

[11] Y. Zheng, C. Song, M.-C. Chen, B. Xia, W. Liu, Q. Guo, L. Zhang, D. Xu, H. Deng, K. Huang et al., Phys. Rev. Lett. 118, 210504 (2017).

[12] S. K. Leyton and T. J. Osborne, arXiv:0812.4423.

[13] D. W. Berry, J. Phys. A: Math. Theor. 47, 105301 (2014).

[14] D. W. Berry, A. M. Childs, A. Ostrander, and G. Wang, Commun. Math. Phys. 356, 1057 (2017).

[15] T. Xin, B.-X. Wang, K.-R. Li, X.-Y. Kong, S.-J. Wei, T. Wang, D. Ruan, and G.-L. Long, Chin. Phys. B 27, 020308 (2018).

[16] I. L. Chuang, N. Gershenfeld, and M. Kubinec, Phys. Rev. Lett. 80, 3408 (1998).

[17] J. A. Jones, M. Mosca, and R. H. Hansen, Nature (London) 393, 344 (1998).

[18] L. M. Vandersypen, M. Steffen, G. Breyta, C. S. Yannoni, M. H. Sherwood, and I. L. Chuang, Nature (London) 414, 883 (2001).

[19] X. Peng, Z. Liao, N. Xu, G. Qin, X. Zhou, D. Suter, and J. Du, Phys. Rev. Lett. 101, 220405 (2008).

[20] I. L. Chuang, L. M. Vandersypen, X. Zhou, D. W. Leung, and S. Lloyd, Nature (London) 393, 143 (1998).

[21] L. M. K. Vandersypen, M. Steffen, G. Breyta, C. S. Yannoni, R. Cleve, and I. L. Chuang, Phys. Rev. Lett. 85, 5452 (2000).

[22] J. Du, N. Xu, X. Peng, P. Wang, S. Wu, and D. Lu, Phys. Rev. Lett. 104, 030502 (2010).
[23] G. Feng, G. Xu, and G. Long, Phys. Rev. Lett. 110, 190501 (2013).

[24] Z. Li, X. Liu, N. Xu, and J. Du, Phys. Rev. Lett. 114, 140504 (2015).

[25] C. Moler and C. Van Loan, SIAM Rev. 45, 3 (2003).

[26] L. Gui-Lu, Commun. Theor. Phys. 45, 825 (2006).

[27] S. Gudder, Quantum Inf. Process. 6, 37 (2007).

[28] A. M. Childs and N. Wiebe, Quantum Inf. Comput. 12, 901 (2012).

[29] S.-J. Wei and G.-L. Long, Quantum Inf. Process. 15, 1189 (2016).

[30] V. Giovannetti, S. Lloyd, and L. Maccone, Phys. Rev. Lett. 100, 160501 (2008).

[31] V. Giovannetti, S. Lloyd, and L. Maccone, Phys. Rev. A 78, 052310 (2008).

[32] J. Biamonte, P. Wittek, N. Pancotti, P. Rebentrost, N. Wiebe, and S. Lloyd, Nature (London) 549, 195 (2017).

[33] G. H. Golub and C. F. Van Loan, Matrix Computations (Johns Hopkins University Press, Baltimore, 2012), Vol. 3.

[34] T. Xin, S.-J. Wei, J. S. Pedernales, E. Solano, and G.-L. Long, Phys. Rev. A 96, 062303 (2017).

[35] D. Lu, T. Xin, N. Yu, Z. Ji, J. Chen, G. Long, J. Baugh, X. Peng, B. Zeng, and R. Laflamme, Phys. Rev. Lett. 116, 230501 (2016).

[36] J. A. Jones, V. Vedral, A. Ekert, and G. Castagnoli, Nature (London) 403, 869 (2000).

[37] T. Xin, S. Huang, S. Lu, K. Li, Z. Luo, Z. Yin, J. Li, D. Lu, G. Long, and B. Zeng, Sci. Bull. 63, 17 (2018).

[38] E. Knill, I. Chuang, and R. Laflamme, Phys. Rev. A 57, 3348 (1998).

[39] D. G. Cory, A. F. Fahmy, and T. F. Havel, Proc. Natl. Acad. Sci. USA 94, 1634 (1997).

[40] C. A. Ryan, C. Negrevergne, M. Laforest, E. Knill, and R. Laflamme, Phys. Rev. A 78, 012328 (2008).

[41] N. Khaneja, T. Reiss, C. Kehlet, T. Schulte-Herbrüggen, and S. J. Glaser, J. Magn. Reson. 172, 296 (2005).

[42] O. Moussa, M. P. da Silva, C. A. Ryan, and R. Laflamme, Phys. Rev. Lett. 109, 070504 (2012).

[43] J.-S. Lee, Phys. Lett. A 305, 349 (2002).

[44] G. M. Leskowitz and L. J. Mueller, Phys. Rev. A 69, 052302 (2004).

[45] D. G. Kleinbaum and M. Klein, Logistic Regression (Springer, Berlin, 2010), pp. 103-127.

[46] C. Shao, Y. Li, and H. Li, J. Syst. Sci. Complexity 32, 375 (2019). 